\title{
Horizontal Residual Mean: Addressing the Limited Spatial Resolution of Ocean Models
}

\author{
Yuehua Li, Trevor McDougall, And Shane Keating \\ School of Mathematics and Statistics, University of New South Wales, Kensington, \\ New South Wales, Australia \\ CASIMIR DE LAVERGNE AND GURVAN MADEC \\ LOCEAN Laboratory, Sorbonne Université-CNRS-IRD-MNHN, Paris, France
}

(Manuscript received 17 April 2019, in final form 25 June 2019)

\begin{abstract}
Horizontal fluxes of heat and other scalar quantities in the ocean are due to correlations between the horizontal velocity and tracer fields. However, the limited spatial resolution of ocean models means that these correlations are not fully resolved using the velocity and temperature evaluated on the model grid, due to the limited spatial resolution and the boxcar-averaged nature of the velocity and the scalar field. In this article, a method of estimating the horizontal flux due to unresolved spatial correlations is proposed, based on the depth-integrated horizontal transport from the seafloor to the density surface whose spatially averaged height is the height of the calculation. This depth-integrated horizontal transport takes into account the subgrid velocity and density variations to compensate the standard estimate of horizontal transport based on staircase-like velocity and density. It is not a parameterization of unresolved eddies, since it utilizes data available in ocean models without relying on any presumed parameter such as diffusivity. The method is termed the horizontal residual mean (HRM). The method is capable of estimating the spatial-correlation-induced water transport in a $1 / 4^{\circ}$ global ocean model, using model data smoothed to $3 / 4^{\circ}$. The HRM extra overturning has a peak in the Southern Ocean of about $1.5 \mathrm{~Sv}\left(1 \mathrm{~Sv} \equiv 10^{6} \mathrm{~m}^{3} \mathrm{~s}^{-1}\right)$. This indicates an extra heat transport of $0.015 \mathrm{PW}$ on average in the same area. It is expected that implementing the scheme in a coarse-resolution ocean model will improve its representation of lateral heat fluxes.
\end{abstract}

\section{Introduction}

The stirring and mixing of tracers by mesoscale eddies in the ocean interior is thought to occur along locally referenced potential density surfaces (Griffies 2004; McDougall and Jackett 2005; McDougall et al. 2014, 2017). The justification for this "epineutral" direction of mesoscale mixing relies on the observation that density overturns in the ocean interior are observed only at small scales $(<1 \mathrm{~m})$ during active three-dimensional turbulence. The mixing due to such small-scale threedimensional turbulence is best understood and parameterized as isotropic turbulent diffusion (although this type of mixing is often called "diapycnal mixing"). The remaining mixing processes in the ocean interior occur along locally referenced potential density surfaces as if

Corresponding author: Yuehua Li, yuehua.li@unsw.edu.au there were no small-scale density overturns (McDougall et al. 2014). This decomposition is justified by ocean observations at the fine and microscales and motivates the standard approach, in oceanographic theory and modeling, of representing mixing of tracers as the sum of epineutral mixing by mesoscale eddies and isotropic mixing by small-scale turbulence.

A key development in modeling ocean mixing was made by Gent and McWilliams (1990). These authors realized that the epineutral diffusion of scalars would be affected by lateral variations of the thickness between pairs of closely spaced isopycnals, and they proposed a parameterization that acted as a sink of gravitational potential energy via the diffusion of this thickness. At the time it was thought that the Gent and McWilliams (1990) parameterization acted in a diabatic manner, increasing the amount of diapycnal mixing. However, Gent et al. (1995) showed that the parameterization 
could be represented as an extra nondivergent velocity and that the total velocity advects ocean tracers in an adiabatic and isohaline manner.

McDougall and McIntosh (2001) subsequently showed that the Gent and McWilliams (1990) procedure was a parameterization of the eddy contribution to the temporal residual mean (TRM) circulation. The concept of residual mean circulation is common in atmospheric science, where the mean circulation is calculated from a zonal average (Andrews and McIntyre 1976). By contrast, the TRM velocity involves temporal averaging at a fixed longitude and latitude. The TRM theory of McDougall and McIntosh (2001) introduced a two-dimensional quasiStokes streamfunction to represent the extra nondivergent advection due to eddies (the quasi-Stokes velocity). The total TRM velocity is then the sum of the Eulerian mean velocity and the eddy-induced quasi-Stokes velocity. McDougall and McIntosh (2001) showed that the product of the lateral diffusivity and the slope of isopycnals used by Gent and McWilliams (1990) can be regarded as a parameterization of the quasi-Stokes streamfunction.

McDougall and McIntosh (2001) also demonstrated an intuitive link between the quasi-Stokes velocity of the TRM circulation (which is based in Cartesian coordinates) and the eddy-induced extra advection caused by thickness-weighted averaging, which is the natural way of averaging in density coordinates. They considered the horizontal transport of seawater denser than the density surface whose time-mean height is the height being considered, and showed the quasi-Stokes velocity corresponds to the contribution of mesoscale eddies to this horizontal transport of seawater. Thus, in TRM theory, eddy effects are implemented in the conservation equation for the scalar variables (such as temperature and salinity) by modifying both the advective velocity and the advected scalar field. This is in contrast to recent work on representing the role of mesoscale eddies in ocean models by parameterizing eddy effects directly in the momentum equation (Young 2012; Maddison and Marshall 2013; Porta Mana and Zanna 2014).

The Gent and McWilliams (1990) parameterization essentially represents the horizontal density flux due to unresolved temporal correlations between temperature (or salinity) and the horizontal velocity. In the same way, unresolved spatial correlations between temperature and horizontal velocity will contribute horizontal density fluxes that should be included in ocean models which carry scalar fields and velocities on a relatively coarse spatial grid. This type of unresolved spatial correlation and its importance for the oceanic meridional heat transport have been discussed by Rintoul and Wunsch (1991). They found that spatial smoothing significantly reduced the estimate of the northward heat flux across $36^{\circ} \mathrm{N}$ in the Atlantic, due to missing spatial correlations between velocity and temperature. Therefore, insufficient spatial resolution in the western boundary currents of geostrophic box inversions or numerical ocean simulations may result in underestimation of the meridional heat flux.

McDougall (1998) considered the effect of spatial resolution limitations on the horizontal transport of seawater that is denser than the isopycnal whose average height is the height being considered. The term horizontal residual mean (HRM) was coined to describe the total velocity that would include the extra advection of seawater of this density class due to the unresolved spatial correlations. McDougall (1998) also proposed an expression for the eddy-induced HRM streamfunction in terms of the vertical and horizontal shears of the resolved horizontal velocity and the resolved-scale slope of density surfaces. Thus, just as the quasi-Stokes advection of the TRM circulation can be regarded as the adiabatic way of including the horizontal density fluxes due to unresolved temporal correlations between temperature and horizontal velocity, so the eddy-induced advection of the HRM circulation can be regarded as the adiabatic way of including the horizontal density fluxes due to unresolved spatial correlations.

The idea proposed by McDougall (1998) was cast in terms of spatial correlations between the velocity and density that had both been temporally averaged. However in practice, the spatial correlations are present at every instant and can also be calculated at each time step when running ocean models. Hence, we calculate and apply the HRM streamfunction to the spatial correlations of the instantaneous velocity and density surfaces at each time step, instead of using temporal mean values.

To calculate the HRM streamfunction, we linearly interpolate the staircase-like velocity and depth functions in order to represent the velocity and density variation within grid boxes. The linearly interpolated velocity is then integrated from the bottom of the ocean up to a certain isopycnal whose spatially averaged height is the height one is considering. The proposed method hence approximates the transport of seawater that is denser than this isopycnal and characterizes the spatial correlations between the velocity and the density surface. An extra velocity can be derived based on the spatial-correlation-induced transport. This extra velocity should be added to the TRM velocity, which is the sum of the Eulerian-mean and temporal-correlation-induced velocities. The total velocity is the velocity with which tracers are advected, and it includes the extra velocity that is induced by spatial correlations between velocity and density.

In this article, we demonstrate the ability of the proposed HRM approach to capture subgrid-scale spatial correlations using a 3D snapshot from a global ocean 
model, the Modular Ocean Model (MOM) 5. We argue that the HRM method improves an ocean model's ability to incorporate contributions from subgrid-scale processes. We further demonstrate that the HRM component shows a peak of around $1.5 \mathrm{~Sv}\left(1 \mathrm{~Sv} \equiv 10^{6} \mathrm{~m}^{3} \mathrm{~s}^{-1}\right) \mathrm{me}$ ridional overturning in the ACC area and a typical $0.015 \mathrm{PW}$ extra heat transport in the same area. These findings indicate how HRM can influence model results in water transport, heat transport and tracer advections. Here, we focus on the theoretical aspects and preliminary diagnostics of the HRM method. The article is organized in the following way. Expressions for HRM theory are presented in section 2. In section 3 we demonstrate that the method of calculating the transport from coarsely resolved model fields gives a good approximation to the corresponding transport of seawater that would be available in a finer-resolution ocean model. In section 4 we diagnose the contribution of the extra nondivergent advection to basin-scale meridional heat and mass transports obtained from a model snapshot. Section 5 justifies the nontapering choice of the HRM method. We summarize our findings in section 6 .

\section{Expressions for the extra nondivergent advection of HRM}

\section{a. The HRM transport and streamfunction}

Instantaneous density surfaces are commonly not aligned with constant pressure, as shown in Fig. 1a. The horizontal line labeled as $z_{0}$ denotes the constant depth at which water transport is calculated. However, the water transport of interest is the transport of water that is denser than the density surface, shown as the shaded area in Fig. 1a. Since the velocity within a grid box also varies, the spatial correlations between the velocity and slope will contribute to the water transport under the instantaneous density surface. Within a grid box, this contribution by the meridional velocity under the density surface can be represented as

$$
\int_{-\Delta x / 2}^{\Delta x / 2} \int_{z_{0}}^{z_{a}(x)} v(x, z) d z d x
$$

where $\Delta x$ is the zonal width of a grid box, $z_{0}$ is the constant pressure/depth at which one calculates the water transport, $z_{a}(x)$ is an approximated height of the instantaneous density surface, and $v$ is the meridional velocity. Note that $z_{a}(x)$ varies with the longitude $x$ and $v(x, z)$ varies with both the longitude $x$ and height $z$ within a grid box.

In $z$-coordinate ocean models, the height and velocity are calculated and stored as step functions, that is, one grid box corresponds to one height and one velocity. To represent subgrid density and velocity variations, we linearly
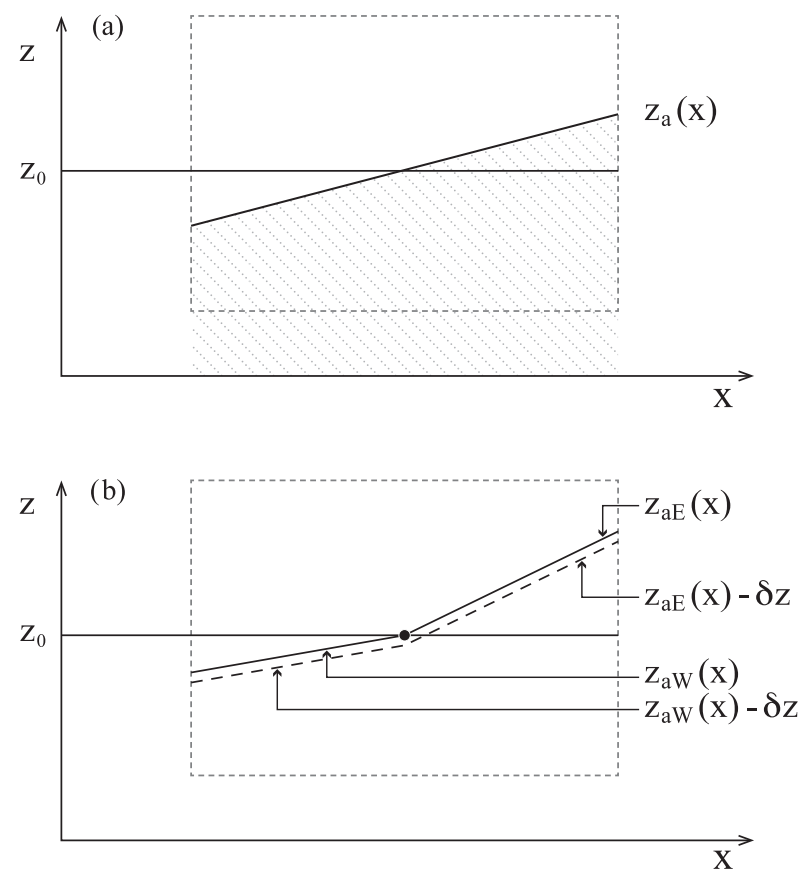

FIG. 1. (a) An illustration of the instantaneous density surface and the constant pressure. The horizontal straight line is the constant pressure $z_{0}$, at which the $z$-coordinate ocean model calculates the water transport within the grid box. The instantaneous density surface $z_{a}(x)$ is illustrated by the straight line sloping up to the right. (b) Slopes on the two sides of a cast are allowed to be different, as shown in Eq. (2). The sloping line in (a) is replaced by two solid straight lines with different slopes. These two slopes can be calculated using available data in ocean models. The HRM theory requires that the density surface has zero average perturbation from $z_{0}$, while the average height of the solid lines is not $z_{0}$. Hence, the transport under the dashed line, $z_{a}(x)-\delta z$, is considered.

interpolate these functions within the grid box. Since the density surface slopes in the model are calculated based on adjacent casts, the density slopes in the two half-boxes of a grid box are typically different, as shown in Fig. 1b. Hence, the instantaneous density height is approximated by two linear approximations, with $z_{a W}(x)$ being the height for the western half and $z_{a E}(x)$ for the eastern half,

$$
z_{a E}(x)=z_{0}+S_{E}^{x} x \text { and } z_{a W}(x)=z_{0}+S_{W}^{x} x,
$$

where $S_{W}^{x}$ and $S_{E}^{x}$ are the western and eastern slopes, respectively. The slope $S^{x}$ is usually calculated and stored in ocean models, thus requiring no additional calculation. The algorithm of calculating the slope $S^{x}$ depends on the ocean model, but usually only requires data from two adjacent casts. The curvature of the density surface as a function of $x$ has been taken into account by allowing $S_{E}^{x}$ and $S_{W}^{x}$ to be different. The two linear approximations are shown in Fig. 1b. The velocity variation within a grid box is approximated by 


$$
v(x, z)=v_{0}+v_{x} x+v_{z}\left(z-z_{0}\right),
$$

where $v_{0}$ denotes the velocity at the center of the grid box, while $v_{x}$ and $v_{z}$ are the zonal and vertical velocity shears, respectively. The way to calculate $v_{x}$ and $v_{z}$ depends on the grid type that the ocean model uses, because the position of velocity varies with different grid types. Substituting linear approximation Eqs. (2) and (3) into Eq. (1), we have

$$
\begin{aligned}
\int_{-\Delta x / 2}^{\Delta x / 2} \int_{z_{0}}^{z_{a}(x)} v(x, z) d z d x= & \frac{1}{8} v_{0}\left(S_{E}^{x}-S_{W}^{x}\right)(\Delta x)^{2} \\
& +\frac{1}{24} v_{x} S_{E}^{x}(\Delta x)^{3}+\frac{1}{24} v_{x} S_{W}^{x}(\Delta x)^{3} \\
& +\frac{1}{48} v_{z}\left(S_{E}^{x}\right)^{2}(\Delta x)^{3}+\frac{1}{48} v_{z}\left(S_{W}^{x}\right)^{2}(\Delta x)^{3} .
\end{aligned}
$$

Notice that the average height of the density surface is not at $z_{0}$, since $S_{E}^{x}$ and $S_{W}^{x}$ are allowed to be different. Rather, the average height differs from $z_{0}$ by

$$
\delta z=\frac{1}{8}\left(S_{E}^{x}-S_{W}^{x}\right) \Delta x .
$$

In the HRM method, we define the spatial average of the density surface height perturbations to be zero. In other words, the average height of the density surface has to be the same as $z_{0}$. Following this definition, the density surface of interest is $z_{a}(x)-\delta z$ rather than $z_{a}(x)$. Hence, the integration should be from $z_{0}$ to $z_{a}(x)-\delta z$. Abovementioned density surfaces $z_{a}(x)$ and $z_{a}(x)-\delta z$ are illustrated in Fig. 1b, with solid lines representing $z_{a}(x)$ [separated into $z_{a E}(x)$ and $z_{a W}(x)$ ] and dashed lines indicating $z_{a}(x)-\delta z$ which has the average height at $z_{0}$. Figure $1 \mathrm{~b}$ demonstrates the case where the average height of $z_{a}(x)$ is above $z_{0}$ ( $\delta z$ is positive). It is also possible for the average of $z_{a}(x)$ to be below $z_{0}$, where $\delta z$ is negative. The meridional HRM transport we consider now is given by

$$
\begin{aligned}
\int_{-\Delta x / 2}^{\Delta x / 2} \int_{z_{0}}^{z_{a}(x)-\delta z} v(x, z) d z d x= & \int_{-\Delta x / 2}^{\Delta x / 2} \int_{z_{0}}^{z_{a}(x)} v(x, z) d z d x+\int_{-\Delta x / 2}^{\Delta x / 2} \int_{z_{a}(x)}^{z_{a}(x)-\delta z} v(x, z) d z d x \\
= & \int_{-\Delta x / 2}^{\Delta x / 2} \int_{z_{0}}^{z_{a}(x)} v(x, z) d z d x-\left(v_{0}-\frac{1}{2} v_{z} \delta z\right) \delta z \Delta x \\
= & \frac{1}{24} v_{x} S_{E}^{x}(\Delta x)^{3}+\frac{1}{24} v_{x} S_{W}^{x}(\Delta x)^{3} \\
& +\frac{1}{48} v_{z}\left(S_{E}^{x}\right)^{2}(\Delta x)^{3}+\frac{1}{48} v_{z}\left(S_{W}^{x}\right)^{2}(\Delta x)^{3} \\
& +\frac{1}{2} v_{z}(\delta z)^{2} \Delta x
\end{aligned}
$$

The zonal HRM transport is similar:

$$
\begin{aligned}
\int_{-\Delta y / 2}^{\Delta y / 2} \int_{z_{0}}^{z_{a}(y)-\delta z} u(y, z) d z d y= & \frac{1}{24} u_{y} S_{N}^{y}(\Delta y)^{3}+\frac{1}{24} u_{y} S_{S}^{y}(\Delta y)^{3} \\
& +\frac{1}{48} u_{z}\left(S_{N}^{y}\right)^{2}(\Delta y)^{3}+\frac{1}{48} u_{z}\left(S_{S}^{y}\right)^{2}(\Delta y)^{3} \\
& +\frac{1}{2} u_{z}(\delta z)^{2} \Delta y
\end{aligned}
$$

where $\Delta y$ is the meridional width of a grid box, $z_{a}(y)$ is the approximated height of the instantaneous density surface in the meridional direction, and $u(y, z)$ is the zonal velocity. Slopes for the north and south half of the grid box are denoted by $S_{N}^{y}$ and $S_{S}^{y}$, respectively. In this direction, 


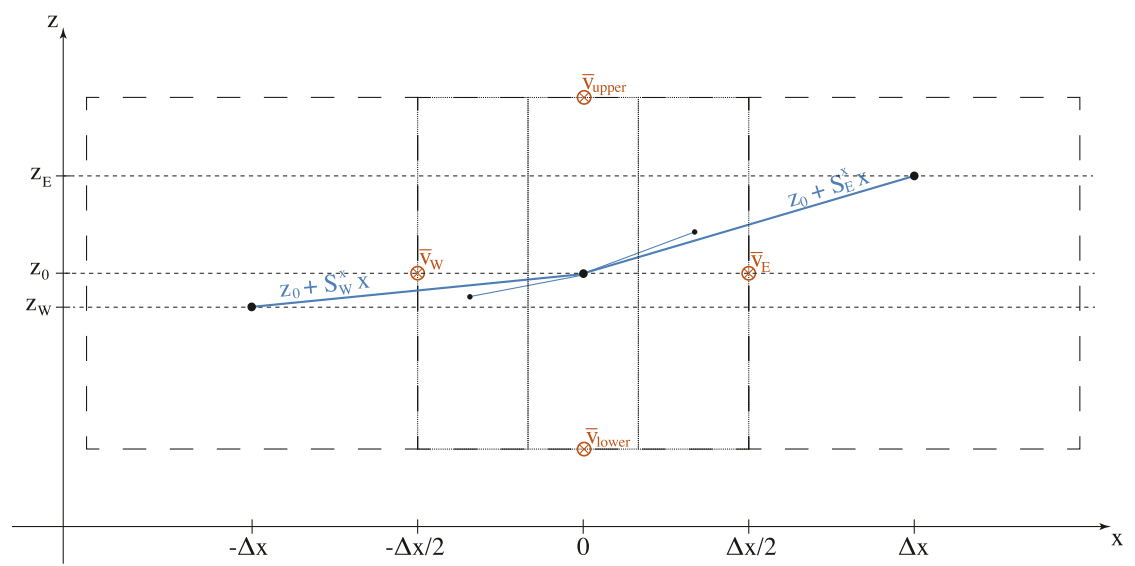

FIG. 2. Vertical cross section through three boxes of a coarse-resolution ocean model, with the central box showing three boxes of a finer-resolution ocean model that has 3 times the horizontal resolution compared with the coarse-resolution model. At the fine-resolution boxes, density surfaces follow the lines from the central point to the small dots at points. The small dots mark intersects of the density surfaces and the tracer casts. At the coarse-resolution, density surfaces follow the lines from the central point to the larger dots. The large dots mark intersects of density surfaces and the tracer casts. The corresponding heights of intersects are denoted $z_{W}$ for the western intersect and $z_{E}$ for the eastern intersect. Since data is on an Arakawa $\mathrm{B}$ grid where velocity is located on the vertices of a grid box, $\bar{v}_{W}$ and $\bar{v}_{E}$ are vertically averaged velocities. For the same reason, $\bar{v}_{\text {upper }}$ and $\bar{v}_{\text {lower }}$ are zonally averaged velocities. The velocities available in the model are originally on vertices of grid boxes.

$$
\delta z=\frac{1}{8}\left(S_{N}^{y}-S_{S}^{y}\right) \Delta y .
$$

The right-hand sides of Eqs. (6) and (7) are expressions for the transport due to unresolved spatial correlations across the width of the boxes of horizontal size $\Delta x$ and $\Delta y$. The corresponding streamfunctions of the extra HRM velocity are found by dividing these equations by $\Delta x$ and $\Delta y$, respectively, so we have

$$
\begin{aligned}
\Psi_{\mathrm{HRM}}^{y}= & \frac{1}{24} v_{x}\left(S_{E}^{x}+S_{W}^{x}\right)(\Delta x)^{2} \\
& +\frac{1}{48} v_{z}\left[\left(S_{E}^{x}\right)^{2}+\left(S_{W}^{x}\right)^{2}+\frac{3}{8}\left(S_{E}^{x}-S_{W}^{x}\right)\right]^{2}(\Delta x)^{2},
\end{aligned}
$$

$$
\begin{aligned}
\Psi_{\mathrm{HRM}}^{x}= & \frac{1}{24} u_{y}\left(S_{N}^{y}+S_{S}^{y}\right)(\Delta y)^{2} \\
& +\frac{1}{48} u_{z}\left[\left(S_{N}^{y}\right)^{2}+\left(S_{S}^{y}\right)^{2}+\frac{3}{8}\left(S_{N}^{y}-S_{S}^{y}\right)^{2}\right](\Delta y)^{2},
\end{aligned}
$$

where we have made use of Eqs. (5) and (8), respectively.

The extra horizontal velocities due to the HRM are then the vertical derivatives of these streamfunctions, with the vertical derivative of $\Psi_{\text {HRM }}^{x}$ [defined in Eq. (10)] being the eastward velocity component, and the vertical derivative of $\Psi_{\mathrm{HRM}}^{y}$ [defined in Eq. (9)] being the northward velocity component.

In a similar way, the extra horizontal quasi-Stokes velocity of the Gent et al. (1995) form of the TRM velocity is the vertical derivative of $\left(\Psi_{\text {TRM }}^{x}, \Psi_{\text {TRM }}^{y}\right)$. On the eastern face of a coarse-resolution box the extra TRM streamfunction is given by $\Psi_{\text {TRM }}^{x}=-\kappa S_{E}^{x}$ while on the northern face it is given by $\Psi_{\text {TRM }}^{y}=-\kappa S_{N}^{y}$. Note that the eastward and northward components of the quasi-Stokes TRM streamfunction are proportional to (minus) the slopes of the density surfaces in these directions. In contrast, the dominance of the first terms in Eq. (9) and (10) (demonstrated in section 3) implies that the eastward component, $\Psi_{\mathrm{HRM}}^{x}$, is proportional to the northward slope of the isopycnals and the northward component, $\Psi_{\mathrm{HRM}}^{y}$, is proportional to the eastward slope of the isopycnals. Thus, the extra advection of HRM and that of TRM act in horizontal directions that are approximately perpendicular to one another.

The streamfunction of the total velocity field is the sum of (i) the Eulerian-mean streamfunction $\left(\bar{\Psi}^{x}, \bar{\Psi}^{y}\right)$, (ii) the quasi-Stokes TRM streamfunction, and (iii) the quasi-Stokes HRM streamfunction, that is,

$$
\begin{aligned}
& \Psi_{\text {Total }}^{x}=\bar{\Psi}^{x}+\Psi_{\mathrm{TRM}}^{x}+\Psi_{\mathrm{HRM}}^{x} \text { and } \\
& \Psi_{\text {Total }}^{y}=\bar{\Psi}^{y}+\Psi_{\mathrm{TRM}}^{y}+\Psi_{\mathrm{HRM}}^{y},
\end{aligned}
$$




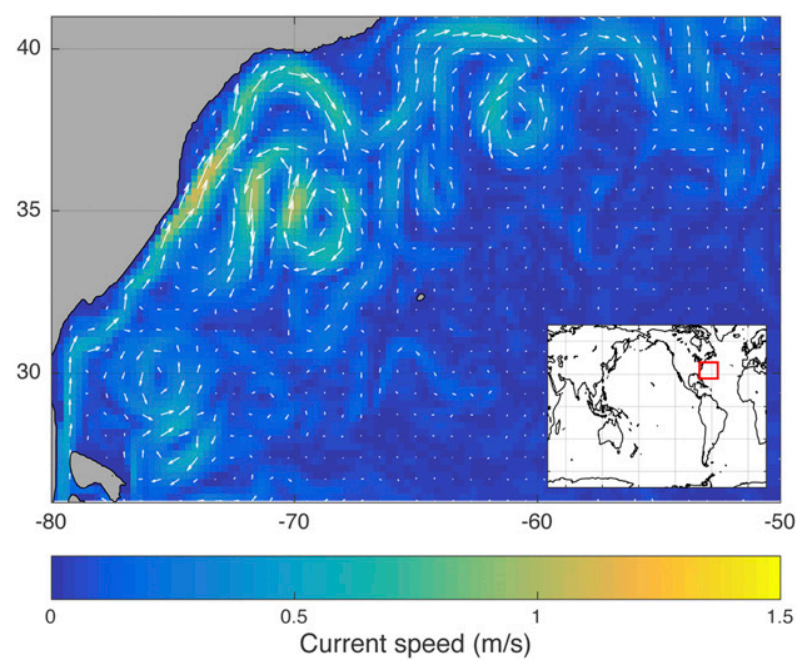

FIG. 3. Fine-resolution current speeds of the Gulf Stream area at a depth of $414 \mathrm{~m}$ are shaded. Velocity arrows are overlain every three grid points.

with the eastward components being evaluated on the eastern face of each coarse-resolution box, and northward component on the northern face of each coarseresolution box. Since the quasi-Stokes streamfunction of the HRM can be readily evaluated using the variables that are available during the running of an ocean model, its adoption should be straightforward. Moreover, the evaluation of this streamfunction can be readily adjusted to the grid on which the model is formulated. This threedimensional quasi-Stokes velocity of the HRM can be added to the Eulerian-mean velocity and the quasi-Stokes velocity of the TRM. The resulting total velocity can be used in ocean models' higher-order advection schemes.

In the following section, we will present details of the HRM transport calculation on an Arakawa B grid ocean model. The contributions of unresolved spatial correlations characterized by HRM equations, namely, Eqs. (6) and (7), can be written in the form of data available in ocean models. We also describe our method for evaluating the accuracy of the contributions from unresolved spatial correlations, using higher-resolution data.

\section{b. Estimating the HRM transport with coarse-resolution data on an Arakawa B grid}

The HRM approach aims at quantifying the unresolved subgrid-scale spatial correlations between velocities and density surfaces [referring to Eqs. (6) and (7)]. These correlations will be calculated within each grid box using data that are already available in ocean models and can complement the subgrid-scale contributions that
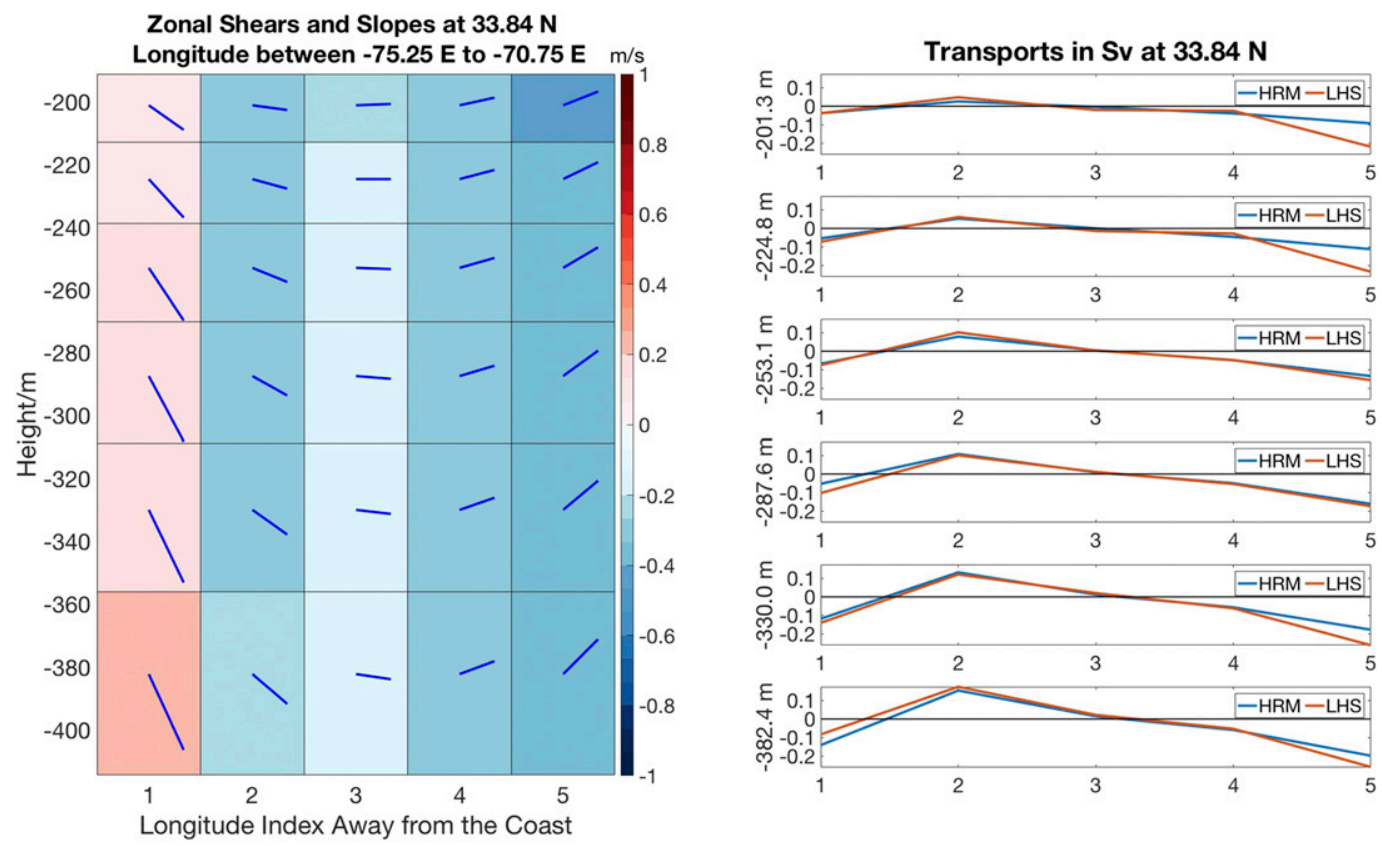

FIG. 4. (left) The zonal velocity differences and slopes in the same selected area as in the right panel. The magnitude of zonal velocity differences is shown as a color map in which red represents positive and blue negative. The averaged slopes of the eastern and western neutral density plane slopes are shown by the blue lines in corresponding grid boxes. (right) The comparison of the transports $\left(\mathrm{Sv} ; 1 \mathrm{~Sv} \equiv 10^{6} \mathrm{~m}^{3} \mathrm{~s}^{-1}\right)$ calculated by the two methods at six different depths in the Gulf Stream region. Red curves correspond to the two-triangle method of the appendix that uses the high-resolution data, while the blue curves correspond to the right-hand side of Eq. (13) applied to the coarse-resolution fields. The $x$ axis is the number of coarse-resolution grid boxes from the coast. 
are not considered at the resolution at which the HRM is implemented. To test the HRM method's ability to compensate the subgrid-scale contributions missed by the current resolution, we calculated the water transport including the HRM transport at a coarse resolution $\left(3 / 4^{\circ}\right.$ in this paper), and then compared it with the transport of water calculated at a finer resolution $\left(1 / 4^{\circ}\right.$ in this paper). The comparison is designed to determine whether the HRM method at the coarse resolution can give a reasonably good approximation to the higher-resolution calculation, in which the $3 / 4^{\circ}$-scale processes are better resolved.

A dataset at $3 / 4^{\circ}$ is constructed by boxcar averaging a snapshot from a $1 / 4^{\circ}$ MOM5 output. The HRM transport is calculated using the $3 / 4^{\circ}$ data and compared with the transport calculated using the $1 / 4^{\circ}$ data. Figure 2 illustrates the coarse- $\left(3 / 4^{\circ}\right)$ and fine-resolution $\left(1 / 4^{\circ}\right)$ grids considered here. The scale of the grid box in the low-resolution model is 3 times that of the fine-resolution grid box, as shown in the central low-resolution box in Fig. 2. The "true" transport on the left-hand side of Eq. (6) is estimated by vertically integrating the velocity data of the fine-resolution model up to the density surface whose heights are also based on the fine-resolution tracer data. This integration of the transport using the fine-resolution model data is described with details in the appendix. The right-hand side of Eq. (6) is calculated using the coarse-resolution data. Slopes are calculated between coarse-resolution casts (see lines labeled as $z_{0}+S_{W}^{x} x$ and $z_{0}+S_{E}^{x} x$ in Fig. 2) and velocities are located on the vertices of coarse-resolution grid boxes in B-grid models. Notice that the velocities used in the calculation $\left(\bar{v}_{W}, \bar{v}_{E}, \bar{v}_{\text {upper }}\right.$ and $\left.\bar{v}_{\text {lower }}\right)$ are not shown on vertices of coarse-resolution grid boxes as they should be. This is because velocities on vertices are spatially averaged, in order to calculate velocity shears $v_{x}$ and $v_{z}$ that apply to the face of each coarse-resolution grid box. More specifically, $\bar{v}_{W}$ and $\bar{v}_{E}$ are the average of velocities at upper and lower vertices of the western and eastern edges of the face, respectively. The terms $\bar{v}_{\text {upper }}$ and $\bar{v}_{\text {lower }}$ are average velocities of adjacent vertices on the upper and lower edges of a grid box face. They are averaged zonally for calculating the vertical shear in Eq. (6) and meridionally for the vertical shear in Eq. (7). In the remainder of this section we describe this procedure in more detail.

On the coarse-resolution model grid, the values of the northward velocity can be estimated at the centers of the eastern, western, upper and lower edges of the northern face of each grid box. In terms of these velocities the velocity shears are $v_{x}=\left(\bar{v}_{E}-\bar{v}_{W}\right) / \Delta x$ and $v_{z}=\left(\bar{v}_{\text {upper }}-\bar{v}_{\text {lower }}\right) / \Delta z$, while the slopes of the density surface are $S_{W}^{x}=$ $\left(z_{0}-z_{W}\right) / \Delta x$ and $S_{E}^{x}=\left(z_{E}-z_{0}\right) / \Delta x$. We take the vertical variation of the northward velocity across the whole width of the box at the height of the density surface to be approximately $v_{z}$ (which is actually the vertical gradient of the northward velocity evaluated at the fixed height $z_{0}$ ). We note again that $z_{W}$ and $z_{E}$ are defined at the centers of the coarseresolution boxes on either side of the central box. Using these expressions, the right-hand side of Eq. (6) can be written in terms of these coarse-resolution model variables as

$$
\int_{-\Delta x / 2}^{\Delta x / 2} \int_{z_{0}}^{z_{a}(x)-\delta z} v d z d x=\frac{1}{24}\left(\bar{v}_{E}-\bar{v}_{W}\right)\left(z_{E}-z_{W}\right) \Delta x+\frac{1}{48} \frac{\left(\bar{v}_{\text {upper }}-\bar{v}_{\text {lower }}\right)}{\Delta z}\left[\left(z_{E}-z_{0}\right)^{2}+\left(z_{W}-z_{0}\right)^{2}+24(\delta z)^{2}\right] \Delta x .
$$

Using Eq. (5) for $\delta z$, Eq. (12) can be rearranged as

$$
\begin{aligned}
\int_{-\Delta x / 2}^{\Delta x / 2} \int_{z_{0}}^{z_{a}(x)-\delta z} v d z d x= & \frac{1}{24}\left(\bar{v}_{E}-\bar{v}_{W}\right)\left(z_{E}-z_{W}\right) \Delta x \\
& +\frac{1}{48} \frac{\left(\bar{v}_{\text {upper }}-\bar{v}_{\text {lower }}\right)}{\Delta z}\left[\left(z_{E}-z_{0}\right)^{2}+\left(z_{W}-z_{0}\right)^{2}+\frac{3}{8}\left(z_{E}+z_{W}-2 z_{0}\right)^{2}\right] \Delta x .
\end{aligned}
$$

Correspondingly, the expression for the extra transport of the HRM in the $x$ direction is

$$
\begin{aligned}
\int_{-\Delta y / 2}^{\Delta y / 2} \int_{z_{a}}^{z_{a}(y)-\delta z} u d z d y= & \frac{1}{24}\left(\bar{u}_{N}-\bar{u}_{S}\right)\left(z_{N}-z_{S}\right) \Delta y \\
& +\frac{1}{48} \frac{\left(\bar{u}_{\text {upper }}-\bar{u}_{\text {lower }}\right)}{\Delta z}\left[\left(z_{N}-z_{0}\right)^{2}+\left(z_{S}-z_{0}\right)^{2}+\frac{3}{8}\left(z_{N}-z_{S}-2 z_{0}\right)^{2}\right] \Delta y,
\end{aligned}
$$




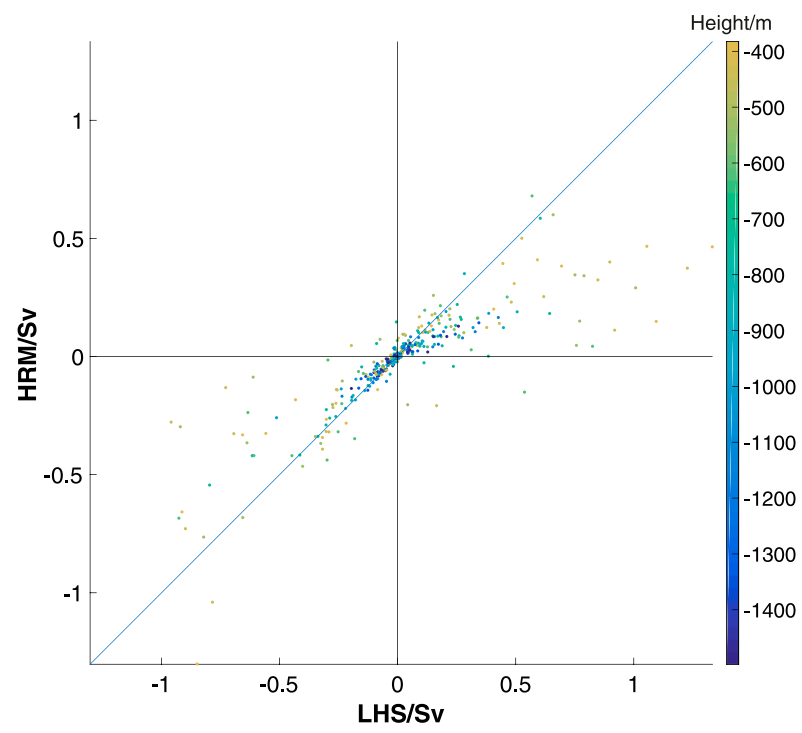

FIG. 5. Scatterplot of transports calculated by the two methods at different latitudes from about $26^{\circ}$ to $41^{\circ} \mathrm{N}$ and different depths from about 382 to $1320 \mathrm{~m}$, in the Gulf Stream. On the $x$ axis is the highresolution estimate of the streamfunction [the left-hand side of Eq. (13)] and on the $y$ axis is the low-resolution estimate [the righthand side of Eq. (13)]. The color bar indicates the heights of the calculated transport in meters.

where $\delta z$ is given by Eq. (8) and can be written as $(1 / 8)\left(z_{N}-z_{0}\right)+(1 / 8)\left(z_{S}-z_{0}\right)$.

In the following section 3 , we will demonstrate results of calculating Eqs. (13) and (14) at $3 / 4^{\circ}$ resolution, using a dataset that was boxcar averaged from the numerical output from the MOM5 ocean model (Griffies 2012) run at a horizontal resolution of $1 / 4^{\circ}$. The results are compared with the calculation at $1 / 4^{\circ}$ in order to demonstrate that the HRM calculations of Eqs. (13) and (14), which rely on the coarse-resolution data, approximately capture the horizontal transport of the fine-resolution model output.

\section{Assessment of the method using $1 / 4^{\circ}$ model snapshot}

We use instantaneous model output from a global MOM5 forced ocean simulation at nominally $1 / 4^{\circ}$ resolution. To construct a low-resolution dataset we boxcar average the model fields over three grid boxes, obtaining a zonal resolution of $3 / 4^{\circ}$. Another way to construct a coarse-resolution dataset from the original data is to subsample over three grid boxes. We have compared these two methods of forming the low-resolution datasets and the results were not significantly different.

The right-hand side of Eq. (13) is an explicit way of calculating the HRM extra transport of water through a

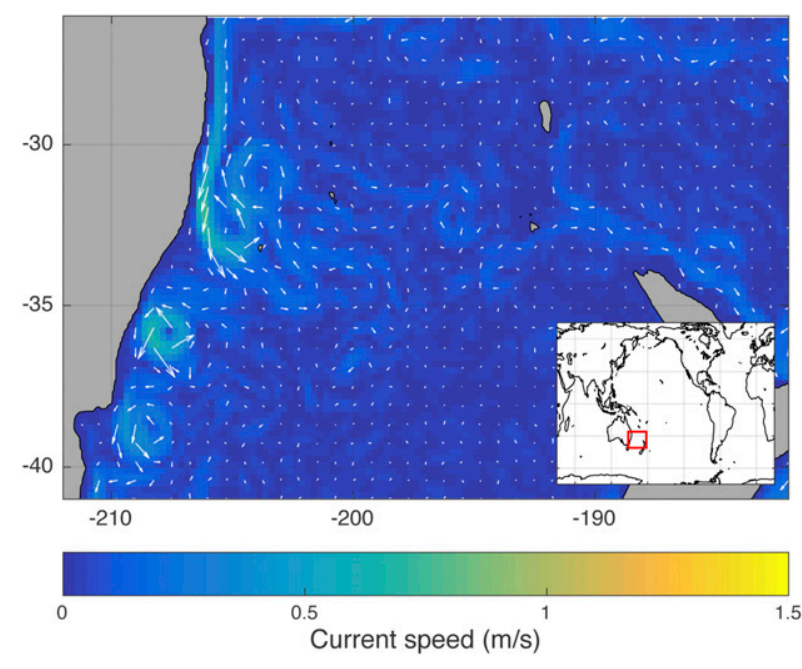

FIG. 6. Fine-resolution current speeds of the East Australian Current area at a depth of $414 \mathrm{~m}$ are shaded. Velocity arrows are overlain every three grid points.

face of a grid box which is centered at height $z_{0}$ and has a zonal width of $\Delta x$. Every value used in this calculation can be obtained after simple and fast operations on the available data from an ocean model. The heights of the density surfaces, namely, $z_{E}, z_{W}, z_{N}$, and $z_{S}$, are calculated on the central cast of the adjacent grid boxes in corresponding directions. Transports are still calculated face by face, and one face in our coarse-resolution calculation is 3 times as large as a face of the fine-resolution calculation that is outlined in the appendix. The other possible way of calculating the HRM extra transport of water is to use slopes of neutral density surfaces, as shown in Eq. (6) using $S_{E}^{x}$ and $S_{W}^{x}$. The terms $S_{E}^{x}$ and $S_{W}^{x}$ are calculated and available to use in some ocean models, such as MOM 6.

The transport of water denser than the density surface whose average height perturbation is zero is given by the left-hand sides of Eqs. (13) and (14). It is evaluated from the fine-resolution model output at $1 / 4^{\circ}$ zonal resolution, using the method described in the appendix. This transport is considered as the true transport and is used as the benchmark for evaluating the ability of the HRM method to approximate the fine-resolution transport while using coarse-resolution data.

In this section we compare these fine-resolution estimates of the volume transport with those produced by the HRM approach applied to the low-resolution data [right-hand sides of Eqs. (13) and (14)]. The comparison is made in three areas: the Gulf Stream, the East Australian Current and the Antarctic Circumpolar Current. In this way, we examine two western boundary current regions as well as the eddy-rich Southern Ocean. We have calculated the quasi-Stokes HRM transports in 

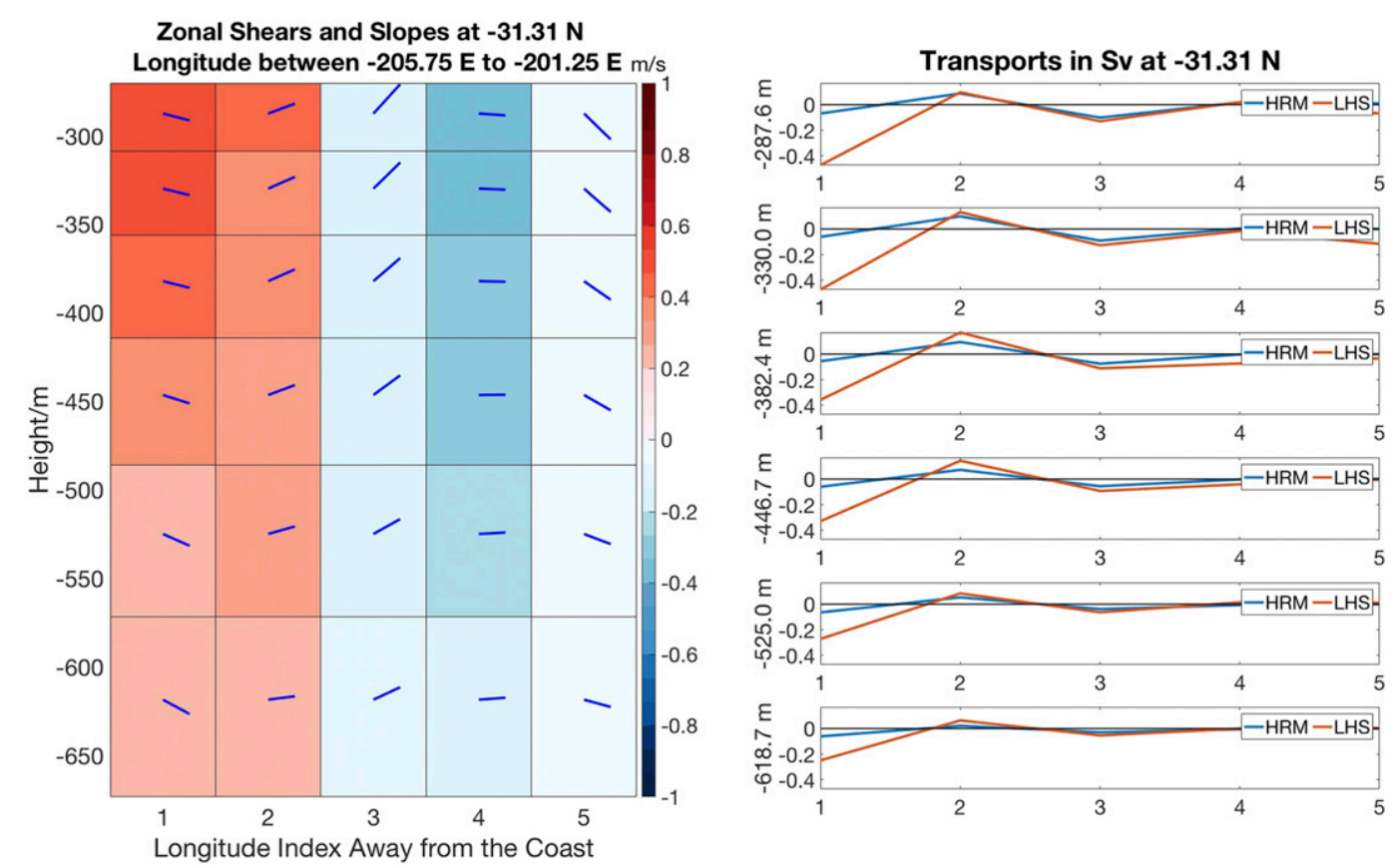

FIG. 7. (left) The zonal velocity differences and slopes in the same selected area as in the right panel. The magnitude of zonal velocity differences is shown as a color map in which red represents positive and blue negative. The averaged slopes of the eastern and western neutral density plane slopes are shown by the blue lines in corresponding grid boxes. (right) The comparison of transport calculated by two methods at six different depths in the East Australia Current. Red curves correspond to the two-triangle method of the appendix that uses the highresolution data, while the blue curves correspond to the right-hand side of Eq. (13) applied to the coarse-resolution fields. The $x$ axis is the number of coarse-resolution grid boxes from the coast.

both the zonal (eastward) and meridional (northward) directions.

\section{a. Gulf Stream}

We examined the region from about $26^{\circ} \mathrm{N}$ to about $41^{\circ} \mathrm{N}$ and from 200 to $1300 \mathrm{~m}$ deep. Figure 3 shows the fineresolution velocity field at a depth of $414 \mathrm{~m}$ to illustrate some features of the chosen area. The underlying colors indicate the fine-resolution current speed and the arrows indicate the fine-resolution velocities, but shown every three grid points to avoid cluttering. Within the Gulf Stream (GS), the northward velocity first increases and then decreases with horizontal distance from the coast.

We compared the values of the transport due to the spatial correlations between estimations using the fine-resolution model output (labeled LHS) and the coarse-resolution output (labeled HRM). The comparisons taken at latitude $33.84^{\circ} \mathrm{N}$ and six successive heights are shown in the right-hand side panel in Fig. 4 for five consecutive coarse-resolution grid boxes starting at the coast. That is, the left-hand-most data points in this figure begin at the first coarse-resolution box adjacent to the coast; these may occur at different longitudes for the different depths shown. This panel demonstrates that the HRM calculation generally gives a good approximation of the true transport due to the spatial correlations, with a tendency to underestimate the true transport that is computed based on the fine-resolution data. The left panel of Fig. 4 demonstrates the magnitude of zonal velocity differences and slopes of the neutral tangent plane across the corresponding grid boxes in the same area as shown in the right-hand side panel. Positive zonal velocity differences are shown as red in the color map and negative ones are blue. Notice that zonal shears have the same sign as zonal velocity differences. The lines emanating from the center of each grid box indicate the directions and magnitudes of the neutral tangent plane slopes. Notice that in Eq. (6), there are two slopes for the eastern and western neutral tangent plane respectively, while in the left panel of Fig. 4, the eastern and western slopes have been incorporated into a single slope for simplicity. The changing sign of these streamfunctions is often caused by the change in sign of the zonal velocity shear $v_{x}$; as already mentioned, the northward velocity first increases and then decreases with distance from the coast. The change of direction of the slope also could result in a change in sign of streamfunctions. The zonal velocity shear and the slope are two dominant factors that affect the streamfunction as discussed later in section 3e. Figure 5 shows a scatterplot comparing these high- and low-resolution 


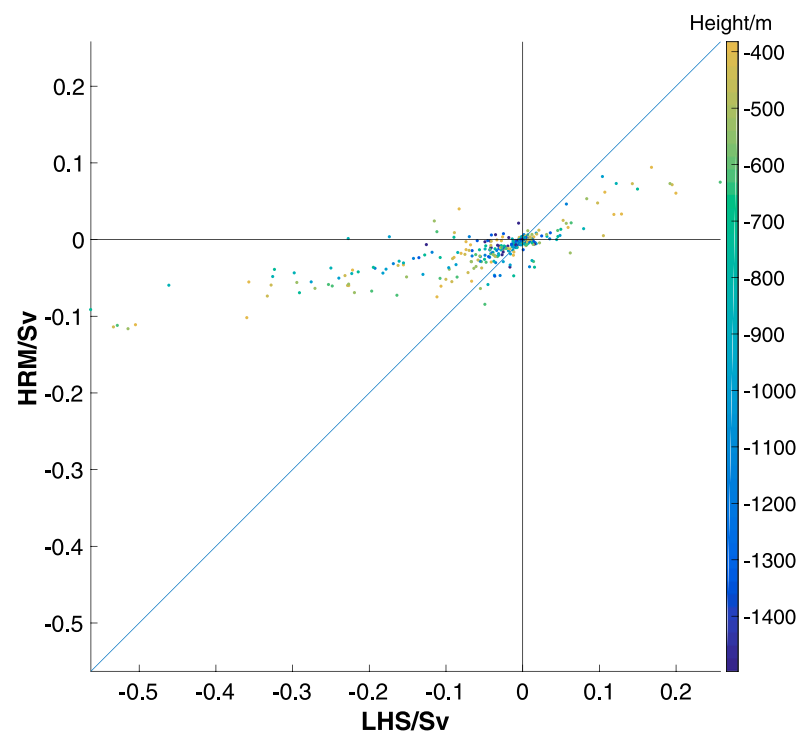

FIG. 8. Scatterplot of transports calculated by the two methods at different latitudes from about $26^{\circ}$ to $41^{\circ} \mathrm{S}$ and different depths from about 382 to $1320 \mathrm{~m}$, in the East Australian Current. On the $x$ axis is the high-resolution estimate of the streamfunction [the left-hand side of Eq. (13)] and on the $y$ axis is the low-resolution estimate [the right-hand side of Eq. (13)]. The color bar indicates the heights of the calculated transport in meters.

estimates of the meridional water transport in the Gulf Stream region. Most of the points stay close to the oneto-one line, but there is a clear indication that the lowresolution estimate of the transport underestimates the true transport by several tens of percentage points. The larger values of the transport occur at relatively shallow depths, while most values at deeper levels are smaller.

\section{b. East Australian Current}

The region chosen for illustrating the transports in the East Australian Current (EAC) is from $26^{\circ}$ to $41^{\circ} \mathrm{S}$ and the same depth range as in the Gulf Stream. A snapshot of the current speed at $414 \mathrm{~m}$ is shown in Fig. 6, with the corresponding comparisons between the fine and coarseresolution estimates of the HRM transport of Eq. (13) shown in Fig. 7 and Fig. 8. The first two columns of grid boxes in Fig. 7 give an example that the changing direction of the slope causes the change in sign of the streamfunction, while the change in sign between the second and the third columns are due to the flipping sign of the zonal velocity shears. Although the coarseresolution-based transport anomaly is generally of the same sign as the fine-resolution one, the former tends to underestimate the latter by about a factor of 2 (referring to Fig. 8). We interpret this underestimation as due to the narrowness of the simulated East Australian Current, which is confined to one or two grid cells along the straight coast, causing partial failure of the HRM

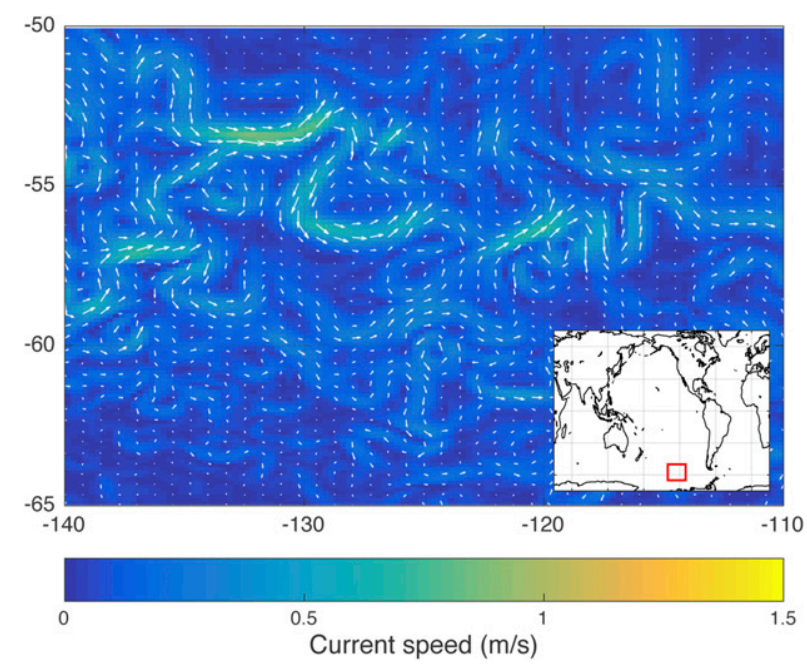

FIG. 9. Fine-resolution current speeds in a region of the Antarctic Circumpolar Current at 414-m depth are shaded. Velocity arrows are overlain every three grid points.

approximation. Hence, in this boundary current region, the extra advection calculated from the coarse-resolution model fields only partly compensates for the missing spatial correlations.

\section{c. Antarctic Circumpolar Current}

We also show a comparison between the left and right hand sides of Eqs. (13) and (14) for a representative subregion in the Antarctic Circumpolar Current (ACC), at latitudes from about $50^{\circ}$ to $65^{\circ} \mathrm{S}$. Figure 9 shows a snapshot of the fine-resolution velocity field of the chosen area at depth of $414 \mathrm{~m}$, illustrating the eddying nature of the ACC. The quasi-Stokes HRM method quite accurately approximates the corresponding transport evaluated using the fine-resolution data, as can be seen in Fig. 10. These favorable results are confirmed in the scatterplots of Figs. 11a and 11b, which show results from all longitudes in the range of latitudes of the ACC.

\section{d. The distribution of estimations}

In previous sections, the scatterplots of fine- and coarse-resolution calculations imply that the HRM method tends to underestimate the true transport. In this section, Fig. 12 plots the distribution of the ratio between coarse- and fine-resolution transport, and hence demonstrates that the trend that the HRM approach underestimates the true transport is dominant in three selected areas described in previous sections and also globally. The first three panels in Fig. 12 show the distribution of the ratio of coarse- to fine-resolution transports in the Gulf Stream, East Australian Current, and Antarctic Circumpolar Current areas, respectively, and the last panel illustrates the global distribution. 

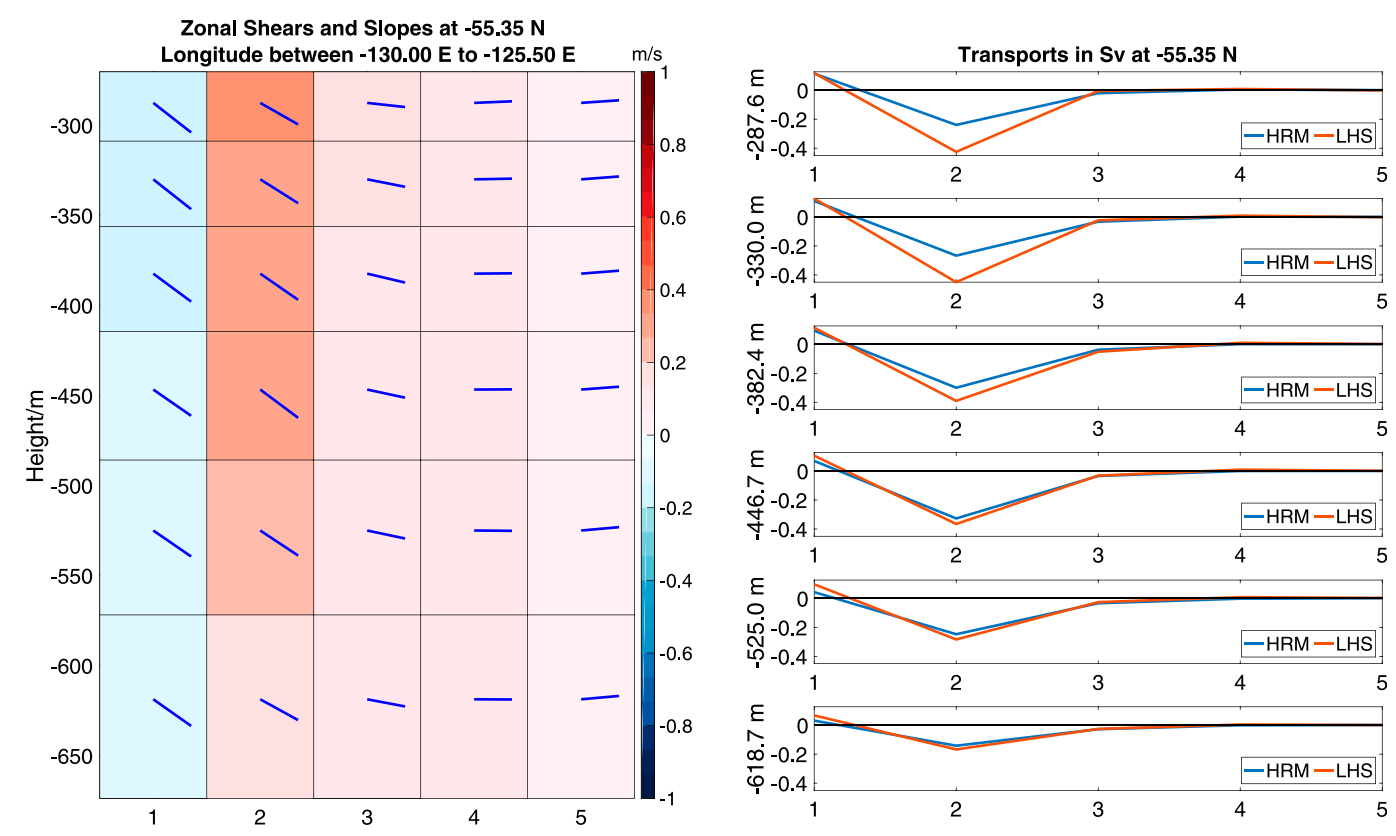

FIG. 10. (left) The zonal velocity differences and slopes in the same selected area as in the right panel. The magnitude of zonal velocity differences is shown as a color map in which red represents positive and blue negative. The averaged slopes of the eastern and western neutral density plane slopes are shown by the blue lines in corresponding grid boxes. (right) The comparison of the transport estimates in the meridional and zonal directions, calculated by two methods at six different depths in the Antarctic Circumpolar Current. Red curves correspond to the high-resolution estimate of the transport, while blue curves correspond the low-resolution estimate. The $x$ axis is number of coarse-resolution grid boxes.

Rather than presenting results at a single depth, ratios plotted in Fig. 12 include calculations at all depths throughout the ocean. Figure 12 excludes ratios where the denominator is within the range of the smallest $20 \%$ of fine-resolution transports, as these small transports are of less interest. However, shapes of the distribution before and after the exclusion are almost identical.

Ratios plotted have been classified into four ranges, namely, from -1.5 to -1 (colored red), from -1 to 0 (colored yellow), from 0 to 1 (colored green), and from 1 to 1.5 (colored blue). The red range is the area we least want the estimation to fall in, because it indicates that the HRM not only has the opposite sign, but also overestimates the true transport. The number of ratios that fall into this bin is much smaller than that in other bins, in each selected area and also globally. The small occurrence of this situation is reassuring. The second bin colored in yellow presents the number of ratios between -1 and 0 , within which the HRM method gives a different sign to the true transport and underestimates the magnitude of the true transport. In all four panels, a relatively large proportion of ratios in this range is very close to 0 . Even though the sign is wrong, the magnitude of HRM transportation is relatively small, which means the impact on the transport will be small as well. In each panel, the range marked green contains the largest proportion of ratios.
Ratios falling in this range demonstrate underestimations with the same sign as the true transport. The results in this range are favorable because they approximate the true transport without imposing the danger of exploding the ocean model. The last blue range gives the numbers of overestimation with the correct sign. Ratios are concentrated within the range from -1 to 1 (yellow and green), predominantly on the positive side, in each selected area and globally. It demonstrates that a large portion of the HRM calculation has the ability to approximate the true transport, with a favorable tendency to underestimate the true transport.

\section{e. The dominance of the horizontal shear term compared with the vertical shear term}

The first term on the right-hand side of Eq. (13) gives the transport induced by the correlation between the zonal variations of velocity and density. In all regions examined we find this first term to be significantly larger than the remaining terms of the equation, which involve the vertical shear of the horizontal velocity. This is illustrated in Fig. 13 and Fig. 14, which show that the horizontal shear term dominates both the meridional and zonal components. Nonetheless, this dominance is less strong in the Gulf Stream region than in the East Australian Current or Antarctic Circumpolar Current regions. 


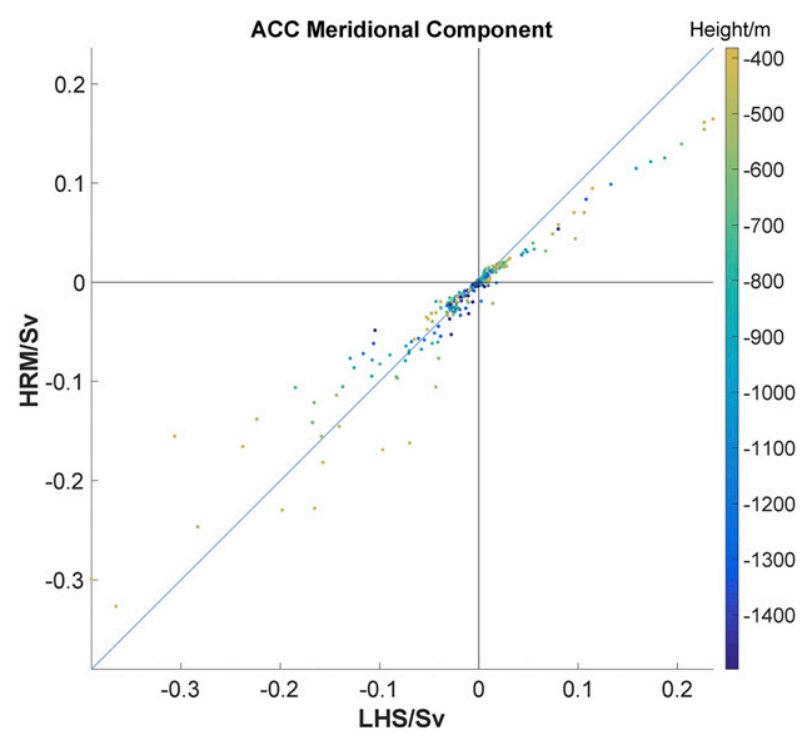

(a)

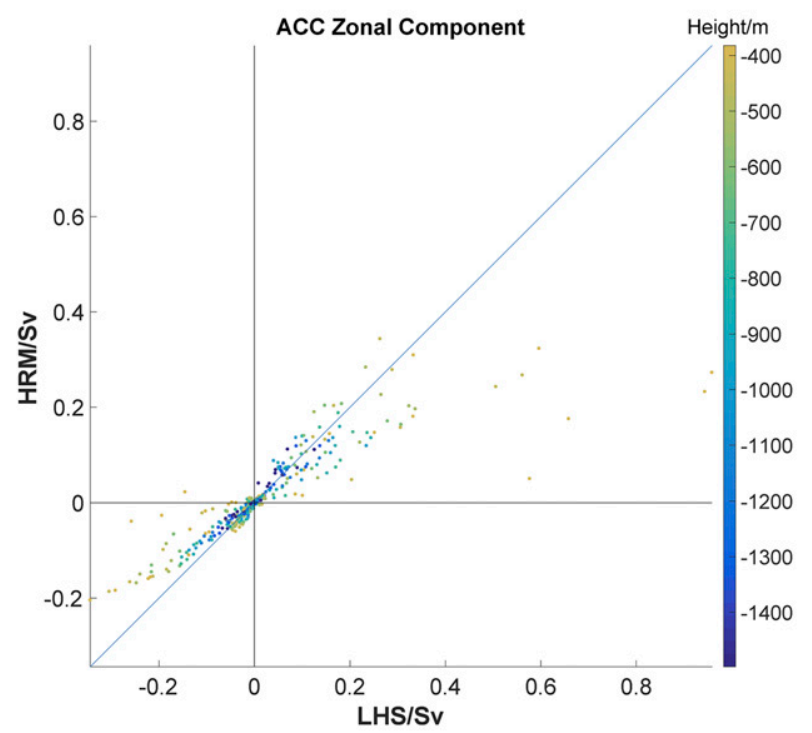

(b)

FIG. 11. Scatterplot of transports calculated by the two methods in the (a) meridional and (b) zonal directions at different latitudes from about $50^{\circ}$ to $65^{\circ} \mathrm{S}$ and different depths from about 382 to $1320 \mathrm{~m}$, in the Antarctic Circumpolar Current. The $x$ axis is the high-resolution estimate of the streamfunction and the $y$ axis is the low-resolution estimate. The color bar indicates the heights of the calculation in meters.

\section{The HRM contribution to meridional overturning and horizontal heat transport}

The contribution of the quasi-Stokes velocity of the HRM to the meridional overturning circulation is estimated by calculating the zonally integrated meridional streamfunction $\Psi_{\text {HRM }}^{y}$ given by Eq. (9). The extra meridional overturning of the HRM is dominated by a cell in the ACC region of strength $1.5 \mathrm{~Sv}$, as shown in Fig. 15. This overturning cell has the same sign and a similar structure to that induced by the advection of the GM scheme as calculated by Gent et al. (1995): it advects surface waters southward and deeper water northward, opposing the Ekman-induced overturning. Nevertheless, at a strength of $1.5 \mathrm{~Sv}$, the extra meridional overturning induced by the HRM method is approximately $10 \%$ of that from the GM presented in Gent et al. (1995), around $12 \%$ of the GFDL-GM calculated in Ferrari et al. (2010) and from $12 \%$ up to $25 \%$ of the proposed parameterization in Ferrari et al. (2010).

Rintoul and Wunsch (1991) compared the heat fluxes calculated by their inverse model with that of a previous study, which used the same data and a similar method. The difference in the magnitude of the heat fluxes calculated by different studies was surprisingly large. Hence they did more experiments and concluded that spatial smoothing was primarily responsible for the difference in the results. The present scheme aims to incorporate the spatial correlations between velocity and scalar quantities that are missing in ocean models, due to the limited spatial resolution and the boxcar-averaged nature of the velocity and the scalar field. In this way, it is expected that implementing the scheme into a coarse-resolution ocean model will improve its representation of lateral heat fluxes. In this section, the meridional heat fluxes induced by the extra HRM advection are calculated and analyzed. The depth-integrated heat fluxes are calculated across the northern and eastern faces of every coarseresolution grid column. Where the face of an individual box is land, the streamfunction there is put equal to zero before performing the vertical integration.

As in McDougall and McIntosh (2001), the contribution of the extra streamfunction to the horizontal heat flux is

$$
\begin{aligned}
\rho_{0} c_{p}^{0} \int_{-H}^{0} \frac{d \Psi_{\mathrm{HRM}}}{d z} \Theta d z & =\rho_{0} c_{p}^{0} \int_{\text {bottom }}^{\mathrm{top}} \Theta d \Psi_{\mathrm{HRM}} \\
& \approx \rho_{0} c_{p}^{0} \sum_{i=1}^{N} \Theta_{i}\left(\Psi_{\mathrm{HRM}, i}-\Psi_{\mathrm{HRM}, i+1}\right),
\end{aligned}
$$

where $\Theta$ stands for Conservative Temperature, $\rho_{0}$ is taken to be $1030 \mathrm{~kg} \mathrm{~m}^{-3}$, and the constant value of the specific heat at constant pressure $c_{p}^{0}$ is the TEOS-10 value given by the Gibbs Seawater (GSW) code (McDougall and Barker 2011). The last step of Eq. (15) is the finite amplitude approximation to the integrals on the first line. The index $i$ in this equation goes from 1 at the sea surface to $N$ at the sea floor. The streamfunction is first interpolated onto the interface heights (the heights of the top and bottom of the model boxes) before being used in this 


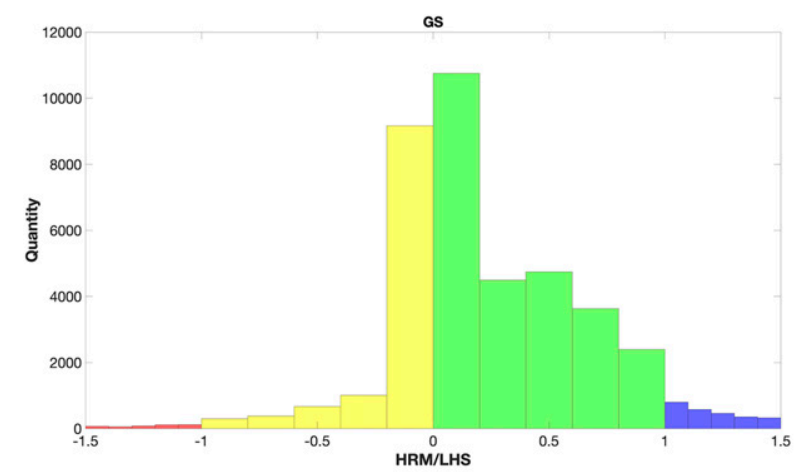

(a)

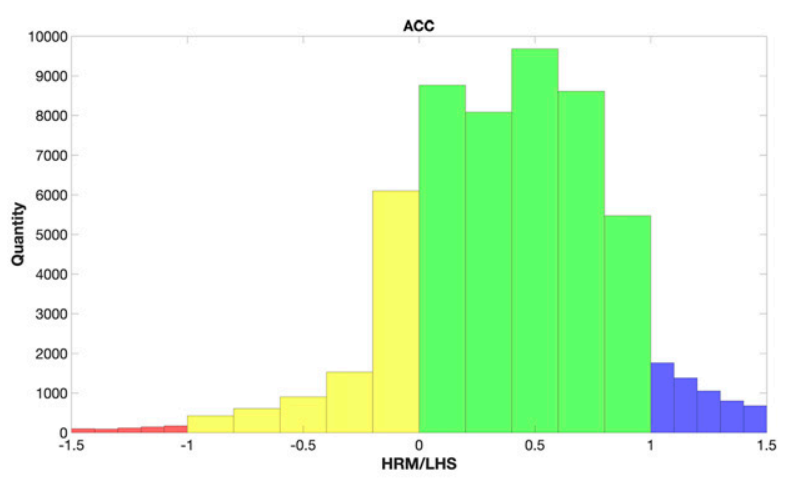

(c)

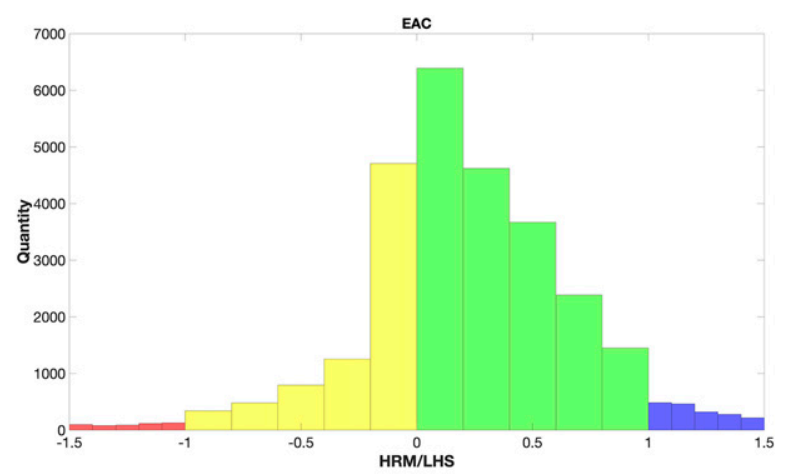

(b)

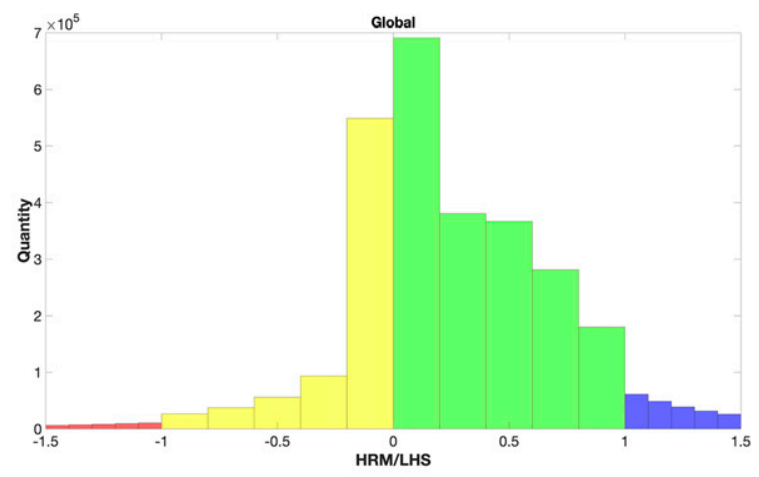

(d)

FIG. 12. The distribution of estimations made by the HRM. The first three panels show the distribution of the ratio of HRM to LHS transports in the same selected areas described in previous areas and the last panel demonstrates the global distribution. Rather than presenting results at a single depth, the ratios plotted include calculations at all depths throughout the ocean. The ratio has been classified into four ranges: from -1.5 to -1 (colored red), from -1 to 0 (colored yellow), from 0 to 1 (colored green), and from 1 to 1.5 (colored blue).

equation. For example, the shallowest box has index $i=1$ with Conservative Temperature $\Theta_{1}$ at the midheight of the grid box and the interpolated HRM streamfunction $\Psi_{\mathrm{HRM}, 1}$ is forced to be zero at the sea surface, and $\Psi_{\mathrm{HRM}, 2}$ is at the bottom of this grid box. The HRM streamfunction at the sea floor $\Psi_{\mathrm{HRM}, N+1}$ is also forced to be zero. This ensures that the vertically integrated mass flux is zero within each water column, so that the depth-integrated extra heat flux given by Eq. (15) is independent of whether the temperature is measured in kelvins or degrees Celsius. The depth-integrated heat flux, $\rho_{0} c_{p}^{0} \sum_{i=1}^{N} \Theta_{i}\left(\Psi_{\mathrm{HRM}, i}-\right.$ $\left.\Psi_{\mathrm{HRM}, i+1}\right)$, is shown in Fig. 16. As expected, the additional heat fluxes introduced by the scheme are concentrated along the coast and at eddying locations, where flows are both strong and of relatively small scale. When the values of the meridional component of Eq. (15) are summed across all longitudes we obtain the contribution of the quasi-Stokes velocity field to the oceanic meridional heat transport. This contribution is typically $0.015 \mathrm{PW}$, and almost $0.02 \mathrm{PW}$ at the latitudes of the ACC in the Southern Ocean (Fig. 17). This 0.02 PW extra HRM heat transport is approximately $10 \%$ the contribution from GM at $60^{\circ} \mathrm{S}$ (Gent et al. 1995). Thus, introducing the HRM extra advection into an ocean model run at $3 / 4^{\circ}$ resolution could impact the simulated meridional heat fluxes. Figure 17 indicates that, when running an ocean model at $3 / 4^{\circ}$ resolution, the HRM correlations that are missing due to the coarse nature of the model grid means that the model misses typically $0.015 \mathrm{PW}$, and misses almost $0.02 \mathrm{PW}$ at the latitudes of the ACC in the Southern Ocean, of meridional heat flux which can readily be added to the model code using our quasi-Stokes HRM streamfunction approach. This is an offline calculation. Caution should be taken when interpreting the implications of the result, since snapshots may include noise. The importance of the extra HRM heat transport remains to be investigated when it is incorporated into forward models.

\section{Tapering of the quasi-Stokes HRM streamfunction}

Our HRM treatment has not applied tapering near the sea surface. At the surface, the intersection of the neutral tangent plane on an adjacent cast may be located 


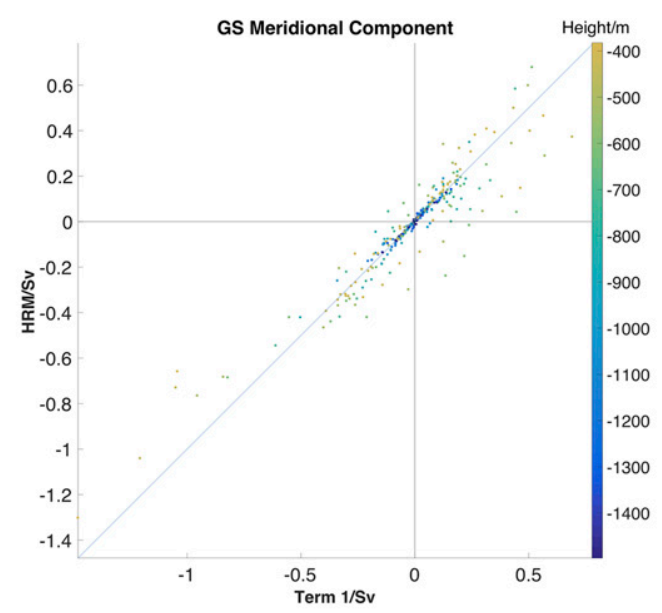

(a)

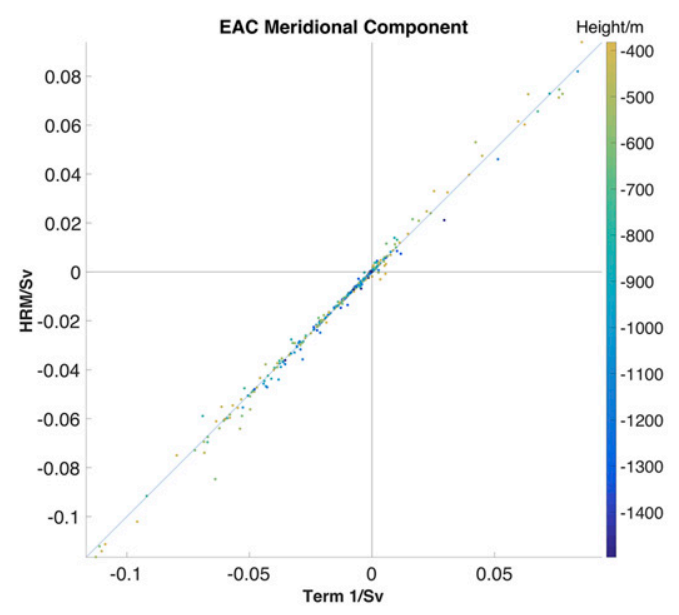

(c)

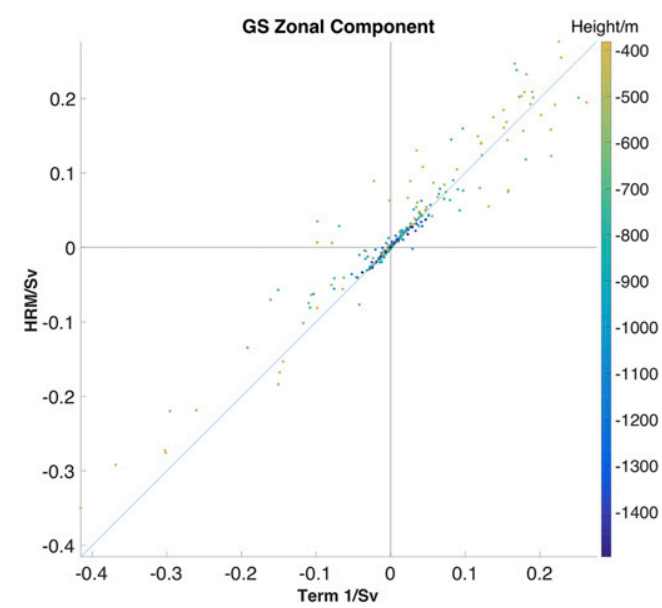

(b)

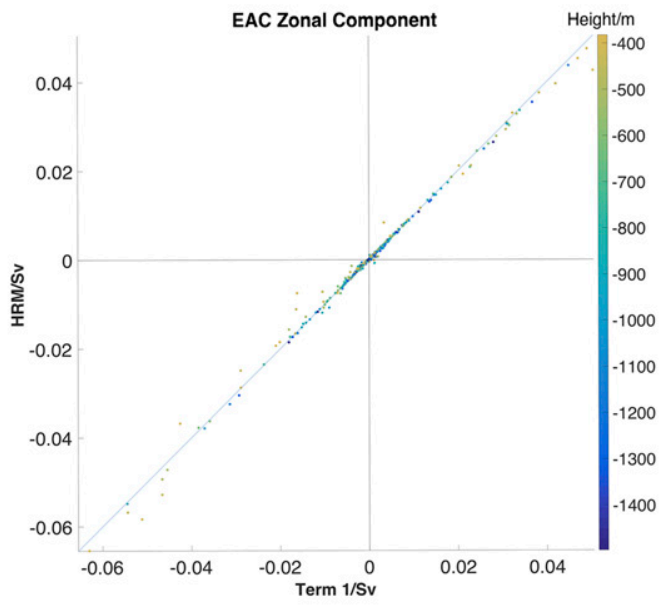

(d)

FIG. 13. The first term (the horizontal shear term) of the right-hand sides of Eqs. (6) (meridional) and (7) (zonal) is plotted on the $x$ axis, with the full right-hand sides of these equations plotted on the $y$ axis of these figures. (a),(b) Comparisons in the selected Gulf Stream region in the meridional and zonal directions, respectively. (c),(d) As in (a) and (b), but for the East Australian Current region. The color bar indicates the corresponding heights at which the terms are calculated.

above the sea level. However, the effective height difference we used is actually half of that calculated on the adjacent cast (see the red dot in Fig. 18a), because each calculation of the HRM transport is the transport through half of the face of a grid box (the eastern, western, northern, or southern half), shown as one of the shaded areas in Fig. 18a. Even if the effective height difference, based on extrapolating a given isopycnal surface, would tend to outcrop as shown in Fig. 18b, our estimate of the HRM streamfunction which clamps the height at the sea surface is an underestimate of the true volume flux. These considerations justify our decision to not taper the HRM streamfunction toward zero except right at the sea surface. This is an important difference between the quasi-Stokes TRM and HRM streamfunctions, since the former uses a gradual taper toward zero at the upper and lower boundaries, which was physically justified by McDougall (1998) and McDougall and McIntosh (2001).

\section{Conclusions}

We have proposed a method of approximating the transport of scalar quantities due to spatial correlations that are unresolved by ocean models. There are three key components of the proposed method. First, the proposed method is based on the widely accepted argument that mixing is mostly along density surfaces. Second, the method applies a linear approximation 


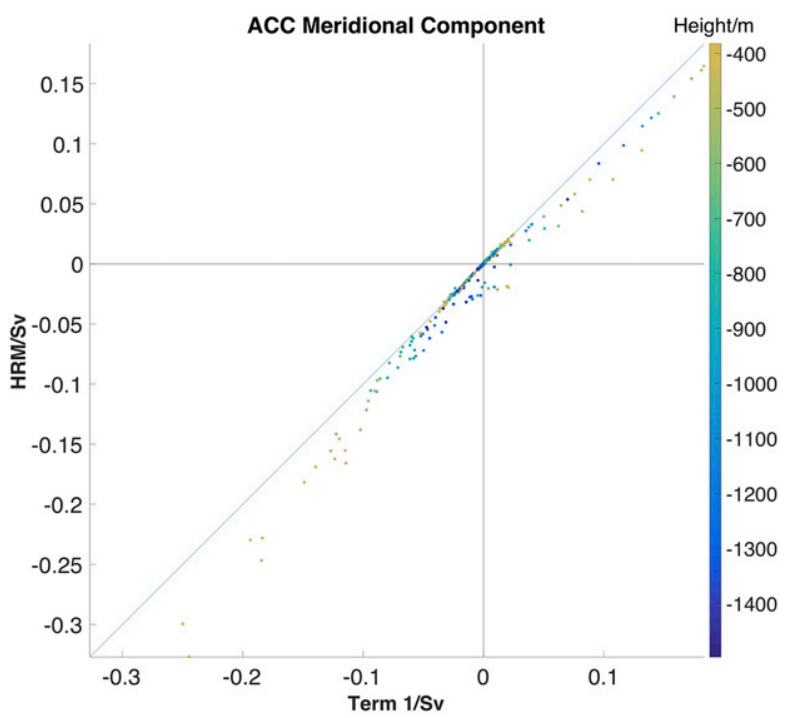

(a)

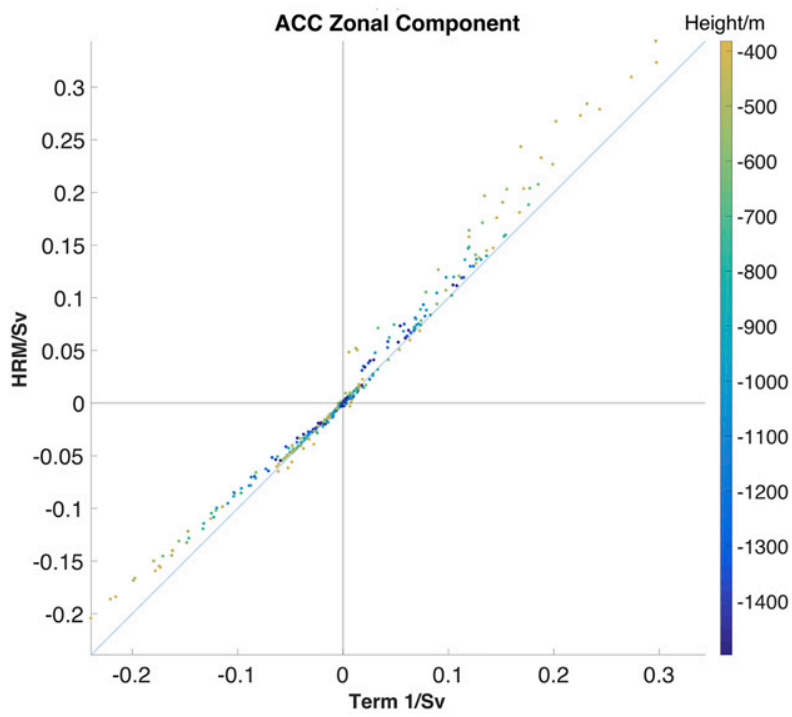

(b)

FIG. 14. The first term (the horizontal shear term) of the righthand sides of equations Eqs. (6) (meridional) and (7) (zonal) is plotted on the $x$ axis, with the full right-hand sides of these equations plotted on the $y$ axis of these figures. The comparison in the (a) meridional direction and (b) zonal direction. The color bar indicates the corresponding heights at which the terms are calculated.

to subgrid velocity and density variations. Third, no parameterization is needed for this method. Our method introduces an extra nondivergent advection, which is calculated from resolved model fields via linear approximations of the spatial variations of the horizontal velocity and the slope of the density surface. This extra advection, or quasi-Stokes HRM velocity, can be added to the Eulerian-mean velocity of the model.

As we have noted, the proposed quasi-Stokes HRM streamfunction does not need a parameterization. Instead, it is estimated directly from the quantities known to the model which appear on the right-hand sides of Eqs. (13) and (14). The HRM captures the unresolved correlations between velocity and density, but does not resolve or parameterize the subgrid-scale physical processes like GM. Therefore, the quasi-Stokes HRM streamfunction should be considered as a complementary and independent component in the total streamfunction, as shown in Eq. (11).

The proposed method has been tested diagnostically using instantaneous output from a $1 / 4^{\circ}$ model simulation, boxcar averaged to $3 / 4^{\circ}$ resolution. We compared the transport of water of a certain density class within the $1 / 4^{\circ}$ dataset to the corresponding HRM extra transport calculated at $3 / 4^{\circ}$ resolution. We found that the method gives a reasonable approximation of the fine-resolution transports in the Gulf Stream, East Australian Current and Antarctic Circumpolar Currents regions, but tends to underestimate the true transport by several tens of percentage points in the first two of these regions. These results suggest that the scheme could assist in mitigating the limitations of coarse-resolution models in the representation of tracer fluxes such as the meridional heat transport.

In the $3 / 4^{\circ}$ resolution dataset, the contribution of the HRM streamfunction to the meridional overturning circulation peaks near $1.5 \mathrm{~Sv}$ in the Southern Ocean, representing about $10 \%$ of the corresponding circulation due to unresolved temporal correlations as parameterized using the Gent et al. (1995) TRM method. The contribution to the poleward heat flux in the Southern Hemisphere of the same dataset reaches $0.02 \mathrm{PW}$. In our discussion of the outcropping of isopycnals at the sea surface, we found no physical reason to taper the quasi-Stokes HRM streamfunction. Indeed, we argued that the outcropping of isopycnals leads to an underestimate of the quasi-Stokes HRM streamfunction.

It may come as a surprise that the zonal integral of the northward quasi-Stokes HRM streamfunction is quite smooth and predominantly of one sign in the ACC region, and that it exhibits a similar structure to the meridional overturning circulation associated with the Gent and McWilliams (1990) parameterization. This similarity may even seem paradoxical when considering that the quasi-Stokes TRM streamfunction, $\left(\Psi_{\mathrm{TRM}}^{x}, \Psi_{\mathrm{TRM}}^{y}\right)=\left(-\kappa S^{x},-\kappa S^{y}\right)$, points in the direction of minus the slope of density surfaces, whereas the quasi-Stokes HRM streamfunction is approximately perpendicular to this direction. However, both the TRM 


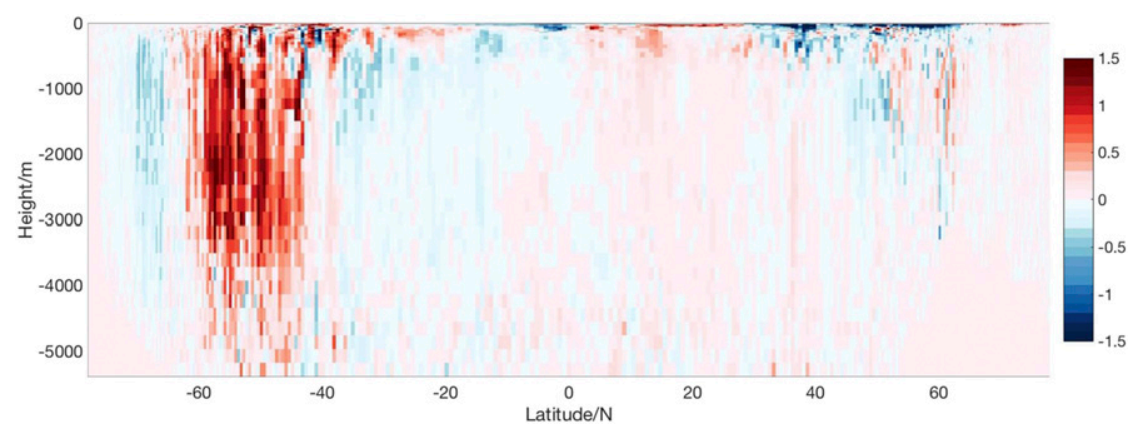

FIG. 15. The meridional overturning streamfunction of the HRM quasi-Stokes velocity in $z$ coordinates. The extra meridional overturning of the HRM is dominated by a cell in the ACC region of strength $1.5 \mathrm{~Sv}$. This overturning cell has the same sign and a similar structure to that induced by the advection of the TRM and calculated with the Gent et al. (1995) scheme: it advects surface waters southward and deeper water northward, opposing the Ekman-induced overturning.

and the HRM quasi-Stokes advection aim to compensate for missing correlations, which, in the context of a modeled $O\left(1^{\circ}\right)$-resolution ACC, arise primarily from the unresolved mesoscale eddies. Both the TRM and the HRM extra streamfunctions thus contribute to mimicking the effect of Southern Ocean mesoscale eddies, which is to oppose the Ekman-forced overturning.

We proposed a method addressing the limited spatial resolution, yet now we ask the question to what extent the HRM method is affected by the resolution itself. According to Eqs. (9) and (10), the HRM velocity streamfunctions are proportional to the second powers of resolution scale $(\Delta x)^{2},(\Delta y)^{2}$. If changing the resolution does not change the velocity shears and neutral density slopes, then the quasi-Stokes HRM transport would decrease proportionally to the fineness of the resolution of the model. However, it is likely that as the horizontal resolution is increased, both the velocity shears and the slope of isopycnals will increase, so it is not yet known how the quasi-Stokes HRM streamfunctions might change as the horizontal resolution is increased. Also, since the HRM method is independent of process-related parameters such as diffusivity, there is no direct indication of how HRM

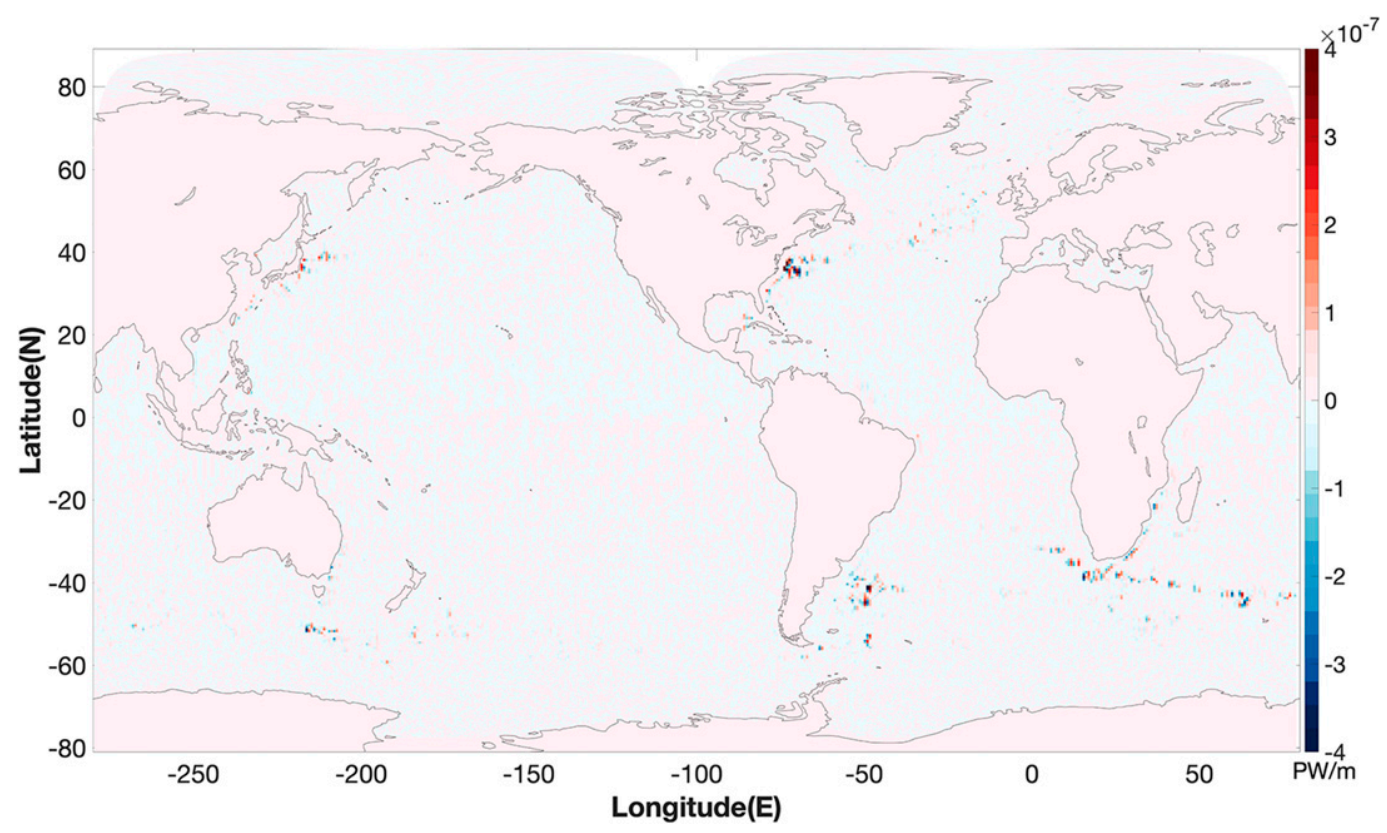

FIG. 16. The global values of the depth-integrated meridional heat flux $\rho_{0} c_{p}^{0} \sum_{i=1}^{N} \Theta_{i}\left(\Psi_{\mathrm{HRM}, i}-\Psi_{\mathrm{HRM}, i+1}\right)$ in $\left(\mathrm{PW} \mathrm{m}{ }^{-1}\right)$. The extra HRM fluxes appear mostly along the cost and in eddy-rich areas. 


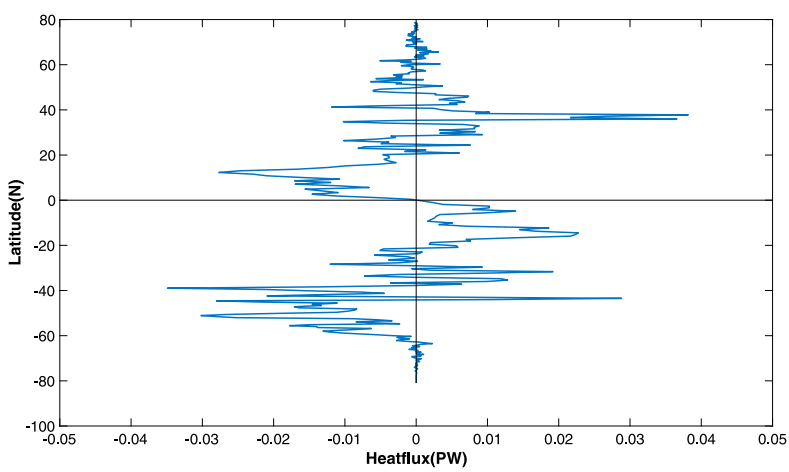

FIG. 17. The quasi-Stokes HRM zonally and depth-integrated meridional heat transport.

would behave as subgrid-scale processes are better resolved.

The HRM streamfunction can be implemented in ocean models to incorporate the contribution from missing spatial correlations. It does not need parameterization and can be calculated using data that are already available in the model. This enhances our confidence in the feasibility of implementing the HRM

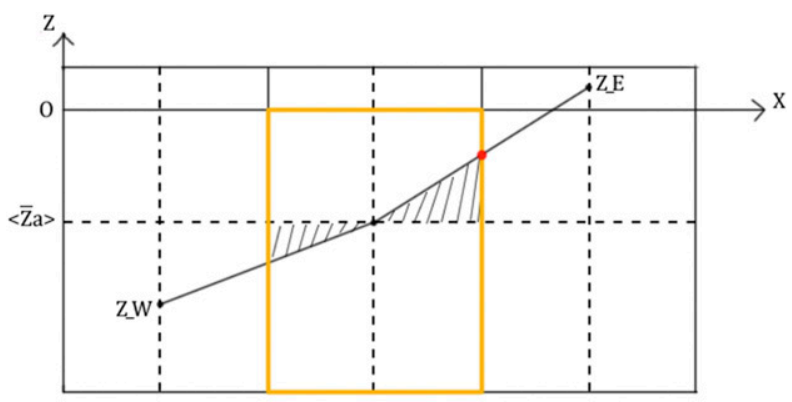

(a)

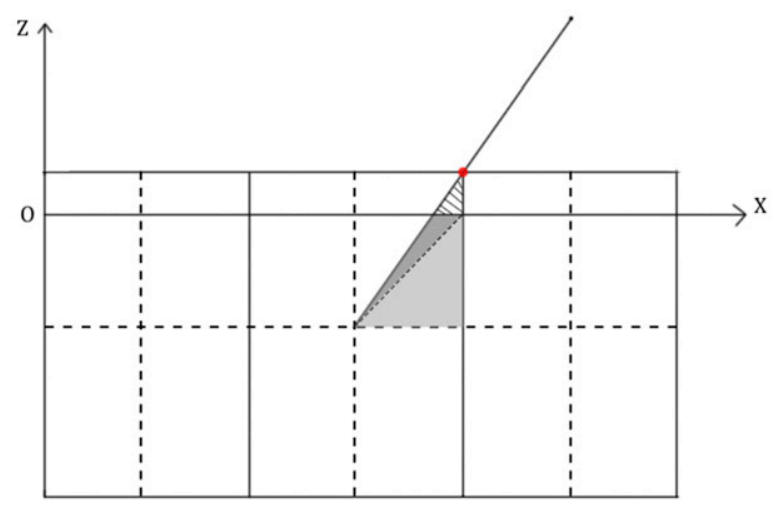

(b)

FIG. 18. An example of outcropping locally referenced neutral tangent plane in which the effective height (the red dot) is below the sea surface.

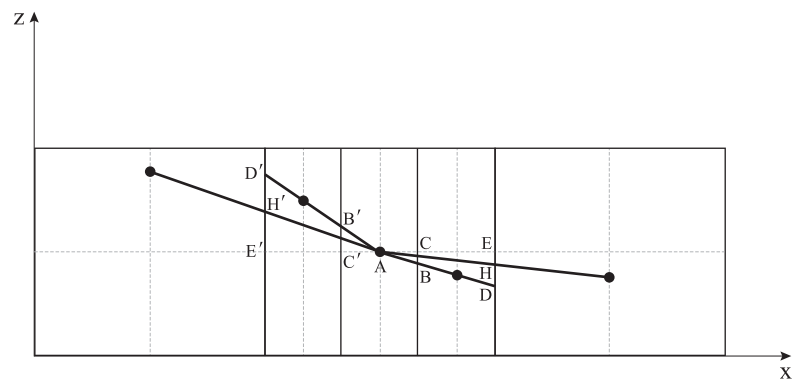

FIG. A1. Vertical cross section through three boxes of a coarseresolution ocean model, with the central box showing three boxes of a finer-resolution ocean model that has 3 times the horizontal resolution compared with the coarse-resolution model. For the fine-resolution boxes, the slopes of the density surfaces are given by the lines from the central point to the dots at points on the finescale grid boxes, while for the coarse-resolution data the slopes of the density surfaces are determined by the lines from the central point to the other two dots at the center (horizontally) of the coarse-resolution boxes.

method into ocean models to capture the unresolved spatial correlations.

Acknowledgments. Louise Bell of Bell Graphic Design (Tasmania) is thanked for preparing Figs. A1 and A2. T. McD and C. de L gratefully acknowledge support from the Australian Research Council through Grant FL150100090. Y. Li acknowledges the support of a University of New South Wales International Postgraduate Award and partial scholarship support from the Australian Research Council Centre of Excellence for Climate System Science (CE110001028) and School of Mathematics and Statistics, UNSW.

\section{APPENDIX}

\section{HRM: Evaluation of the Left-Hand Side of Eq. (6)}

To calculate the left-hand side of Eq. (6), we use a two-triangle calculation. The vertical face at constant latitude through which the transport passes is shown in Fig. A1, and the words "two triangle" refer to triangles $\mathrm{ABC}$ and $\mathrm{ADE}$ for the calculation of transport through area ADE. Figure A1 covers the width of three boxes of the coarse-resolution model, that is, it contains three $T$, $S$ points and eight velocity points. The total transport through the whole area is the sum of the signed transport through $\mathrm{ADE}$ and $\mathrm{AD}^{\prime} \mathrm{E}^{\prime}$ compared with that of the Eulerian-mean transport. Note that because the slopes of $\mathrm{AD}$ and $\mathrm{AD}^{\prime}$ are being calculated separately, they are not necessarily the same. The first step of the twotriangle calculation is to calculate the velocities at points $\mathrm{E}, \mathrm{C}, \mathrm{E}^{\prime}$, and $\mathrm{C}^{\prime}$ by vertically averaging the given velocity 
data that is at the vertices of the cubes of the $T, S$ boxes of the fine-resolution data. Then we calculate the spatially averaged Eulerian velocity at $z_{0}$ using $v_{0}=(1 / 6) v_{\mathrm{E}^{\prime}}+(1 / 3) v_{\mathrm{C}^{\prime}}+(1 / 3) v_{\mathrm{C}}+(1 / 6) v_{\mathrm{E}}$. This spatially averaged Eulerian mean velocity is then subtracted from all velocities to obtain the perturbation velocities. Since the same method is conducted similarly on the western half of Fig. A1 as on the eastern half, we concentrate here on describing what we do on the eastern half.

The heights of points $\mathrm{D}$ and $\mathrm{B}$ are given by $z_{\mathrm{D}}-z_{\mathrm{E}}=$ $(3 / 2)\left(z_{\mathrm{H}}-z_{\mathrm{E}}\right)$ and $z_{\mathrm{B}}-z_{\mathrm{E}}=(1 / 2)\left(z_{\mathrm{H}}-z_{\mathrm{E}}\right)$, where $z_{\mathrm{H}}$ indicates the height where the neutral tangent plane connects the central point A to point $\mathrm{H}$ on the vertical $T$, $S$ cast at the longitude midway between the longitudes of points $\mathrm{C}$ and $\mathrm{E}$. Now knowing the locations of points
$\mathrm{B}$ and $\mathrm{D}$, we find the vertically adjacent locations on the fine-resolution model grid where the velocity components are stored, and the velocities at points B and D are then found by vertical interpolation.

First consider the (perturbation) transport into the page passing through the vertical area ACB. The perturbation velocities at these points are $v_{\mathrm{A}}^{\prime}, v_{\mathrm{C}}^{\prime}$, and $v_{B}^{\prime}$. At any position $(x, z)$ within $\mathrm{ABC}$, the velocity through the vertical area can be denoted as $v^{\prime}=v_{\mathrm{A}}^{\prime}+\left(v_{\mathrm{C}}^{\prime}-v_{\mathrm{A}}^{\prime}\right)(x / X)+$ $\left(v_{\mathrm{B}}^{\prime}-v_{\mathrm{C}}^{\prime}\right)(z / Z)$, where $X$ and $Z$ are the signed lengths of $A C$ and BC. The required horizontal volume flux of marked fluid is equal to the "volume" of a threedimensional space where the spatial directions to the east and upward $(x, z)$ are two of the dimensions, and the third dimension is the perturbation meridional velocity $v^{\prime}$. The volume is

$$
\begin{aligned}
& \text { "volume" of } \mathrm{ABC} \\
& =\int_{0}^{X} \int_{0}^{\frac{x}{X} Z} v^{\prime} d z d x \\
& =\int_{0}^{X} \int_{0}^{\frac{x}{X} Z}\left[v_{\mathrm{A}}^{\prime}+\left(v_{\mathrm{C}}^{\prime}-v_{\mathrm{A}}^{\prime}\right) \frac{x}{X}+\left(v_{\mathrm{B}}^{\prime}-v_{\mathrm{C}}^{\prime}\right) \frac{z}{Z}\right] d z d x \\
& =\int_{0}^{X}\left[v_{\mathrm{A}}^{\prime} \frac{Z}{X} x+\frac{\left(v_{C}^{\prime}-v_{\mathrm{A}}^{\prime}\right)}{X} \frac{Z}{X} x^{2}+\frac{1}{2} \frac{\left(v_{B}^{\prime}-v_{\mathrm{C}}^{\prime}\right)}{Z}\left(\frac{Z}{X}\right)^{2} x^{2}\right] d x \\
& =\frac{1}{2} X Z\left[\frac{1}{3}\left(v_{\mathrm{A}}^{\prime}+v_{\mathrm{B}}^{\prime}+v_{\mathrm{C}}^{\prime}\right)\right] .
\end{aligned}
$$

We note from this expression that the transport into the page is equal to the signed area of triangle $\mathrm{ABC}$ multiplied by the average of the perturbation velocity at the three vertices of the triangle. The derivation of this expression follows the same linear approximation and spatial integration as performed in section 2, and the correspondence to the main HRM result Eq. (13), can be seen as follows. HRM took the perturbation velocity at the center, $v_{\mathrm{A}}^{\prime}$, to be zero, and in this case, we can write the last line of Eq. (A1) as $(1 / 6) X Z\left[2 v_{\mathrm{C}}^{\prime}+\left(v_{\mathrm{B}}^{\prime}-v_{\mathrm{C}}^{\prime}\right)\right]$. With $X$ being half the box width, that is, $X=(1 / 2) \Delta x$, with $Z$ being $Z=(1 / 2) \Delta x S^{x}$, with $2 v_{\mathrm{C}}^{\prime}$ being $v_{x} \Delta x$, and with $\left(v_{\mathrm{B}}^{\prime}-v_{\mathrm{C}}^{\prime}\right)$ being $(1 / 2) S^{x} v_{z} \Delta x$, the right-hand side of Eq. (A1) is one-half of the right-hand side of Eq. (6); the factor of one-half being due to the fact that triangle $\mathrm{ABC}$ represents just the right-hand half of the transport of marked fluid in this model box.

We note that in Eq. (A1), $v_{\mathrm{C}}^{\prime}$ and $v_{\mathrm{B}}^{\prime}$ are not individually important; rather it is their mean value that enters this expression. We will use this property to simplify the evaluation of the three-dimensional space corresponding to area AED, where we will take the average of the perturbation velocities at points $\mathrm{D}$ and $\mathrm{E}$, as well as those at points B and C. In Fig. A2 we sketch the threedimensional volume whose volume we seek to evaluate. We have drawn Fig. A2 with both $v_{\mathrm{D}}^{\prime}$ and $v_{\mathrm{E}}^{\prime}$ equal to the same value, $0.5\left(v_{\mathrm{D}}^{\prime}+v_{\mathrm{E}}^{\prime}\right)$. The same is done for $v_{\mathrm{A}}^{\prime}$ and $v_{\mathrm{B}}^{\prime}$, both having the value $0.5\left(v_{\mathrm{A}}^{\prime}+v_{\mathrm{B}}^{\prime}\right)$. These average perturbation velocities are now used to extrapolate these velocities to the spatial location of point A, obtaining, namely, $v_{\mathrm{A}^{\prime}}^{\prime}$. Note that this extrapolated velocity is different to the actual perturbation velocity at point A, namely, $v_{\mathrm{A}}^{\prime}$ (obtained by interpolation of the perturbation velocities at the height of point $\mathrm{A}$ ).

From Fig. A2 the transport through the vertical triangle ACEDBA of Fig. A1 is equal to the difference between two volumes; being the volume from the $v^{\prime}=0$ plane up to the inclined triangle $\mathrm{A}^{\prime} \mathrm{DE}$, minus the volume between the two inclined triangles $\mathrm{A}^{\prime} \mathrm{BC}$ and $\mathrm{ABC}$. Both of these volumes can be evaluated using the above "triangular volume" equation with suitable reassignments of the corners of the triangle. Note that the first volume 


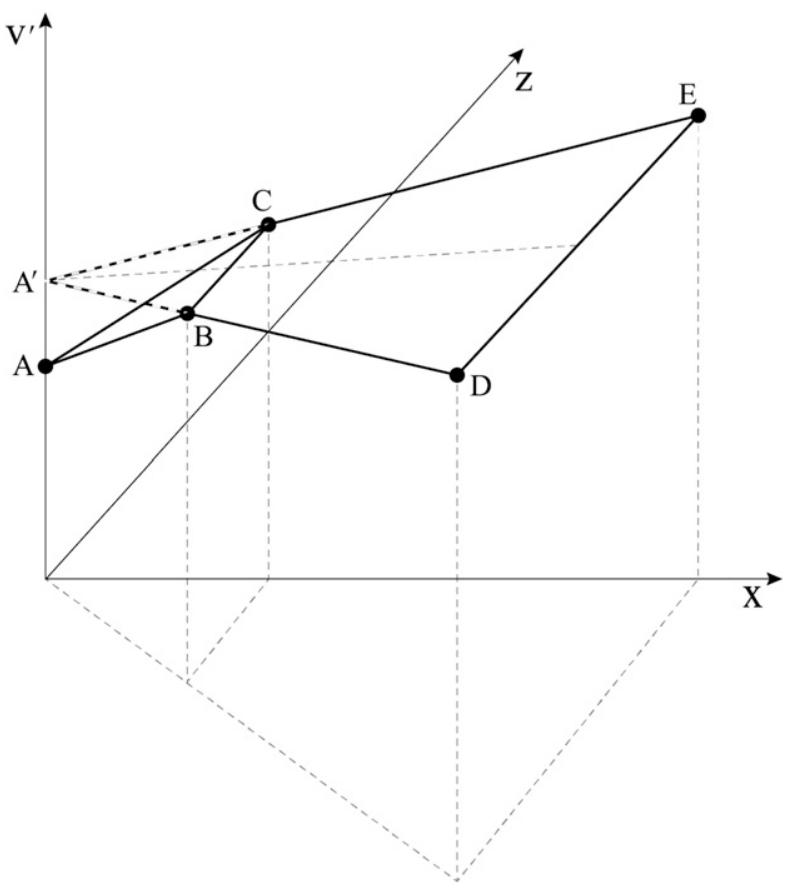

FIG. A2. The three-dimensional view of two-triangle calculation for transports.

usually dominates: for example, the relevant value of $\mathrm{XZ}$ for the large triangle is 9 times the corresponding value of $\mathrm{XZ}$ for the small triangle.

The evaluation of HRM transport is at the average height of the neutral density surface $z_{0}$. However, in practice, the average depth of the above triangle calculations is not necessarily the same as $z_{a}$, since the slopes of the density surfaces are different to the east and to the west. The two-triangle calculation includes extra transport due to its density surface being higher in the water column by the height difference given by Eq. (5), namely, $\delta z=(1 / 8)\left(z_{E}-z_{a}\right)+(1 / 8)\left(z_{W}-z_{a}\right)=$ $(1 / 8)\left(S_{E}^{x}-S_{W}^{x}\right) \Delta x$. The extra transport is

$$
\left(\frac{1}{6} v_{\mathrm{D}^{\prime}}^{\prime}+\frac{1}{3} v_{\mathrm{B}^{\prime}}^{\prime}+\frac{1}{3} v_{B}^{\prime}+\frac{1}{6} v_{D}^{\prime}-\frac{1}{2} v_{z} \delta z\right) \Delta x \delta z,
$$

and this transport is subtracted from that calculated using the above two-triangle calculation.

\section{REFERENCES}

Andrews, D. G., and M. E. McIntyre, 1976: Planetary waves in horizontal and vertical shear: The generalized Eliassen-Palm relation and the mean zonal acceleration. J. Atmos. Sci., 33, 2031-2048, https://doi.org/10.1175/1520-0469(1976)033<2031: PWIHAV $>2.0 . \mathrm{CO} ; 2$.

Ferrari, R., S. M. Griffies, A. J. Nurser, and G. K. Vallis, 2010: A boundary-value problem for the parameterized mesoscale eddy transport. Ocean Modell., 32, 143-156, https://doi.org/ 10.1016/j.ocemod.2010.01.004.

Gent, P. R., and J. C. McWilliams, 1990: Isopycnal mixing in ocean circulation models. J. Phys. Oceanogr., 20, 150-155, https://doi.org/10.1175/1520-0485(1990)020<0150:IMIOCM > 2.0. $\mathrm{CO} ; 2$

—_, J. Willebrand, T. J. McDougall, and J. C. McWilliams, 1995: Parameterizing eddy-induced tracer transports in ocean circulation models. J. Phys. Oceanogr., 25, 463-474, https://doi.org/10.1175/1520-0485(1995)025<0463:PEITTI> 2.0.CO;2.

Griffies, S. M., 2004: Fundamentals of Ocean Climate Models. Princeton Press, $528 \mathrm{pp}$.

_ 2012: Elements of the Modular Ocean Model (MOM): 2012 release. GFDL Ocean Group Tech. Rep. 7, 632 pp., https:// mom-ocean.github.io/assets/pdfs/MOM5_manual.pdf .

Maddison, J. R., and D. P. Marshall, 2013: The Eliassen-Palm flux tensor. J. Fluid Mech., 729, 69-102, https://doi.org/10.1017/ jfm.2013.259.

McDougall, T. J., 1998: Three-dimensional residual-mean theory. Ocean Modeling and Parameterization, E. P. Chassignet and J. Verron, Eds., Springer, 269-302, https://doi.org/10.1007/97894-011-5096-5_12.

—_, and P. C. McIntosh, 2001: The temporal-residual-mean velocity. Part II: Isopycnal interpretation and the tracer and momentum equations. J. Phys. Oceanogr., 31, 1222-1246, https://doi.org/10.1175/1520-0485(2001)031<1222:TTRMVP> 2.0.CO;2.

— and D. R. Jackett, 2005: The material derivative of neutral density. J. Mar. Res., 63, 159-185, https://doi.org/10.1357/ 0022240053693734.

_ , and P. M. Barker, 2011: Getting started with TEOS-10 and the Gibbs Seawater (GSW) Oceanographic Toolbox. SCOR/IAPSO WG127, 28 pp., http://www.teos-10.org/pubs/ Getting_Started.pdf.

— S. Groeskamp, and S. M. Griffies, 2014: On geometrical aspects of interior ocean mixing. J. Phys. Oceanogr., 44, 2164 2175, https://doi.org/10.1175/JPO-D-13-0270.1.

McDougall, T., S. Groeskamp, and S. Griffies, 2017: Comment on Tailleux, R. Neutrality versus materiality: A thermodynamic theory of neutral surfaces. Fluids 2016, 1, 32. Fluids, 2, 19, https://doi.org/10.3390/fluids2020019.

Porta Mana, P., and L. Zanna, 2014: Toward a stochastic parameterization of ocean mesoscale eddies. Ocean Modell., 79, 120, https://doi.org/10.1016/j.ocemod.2014.04.002.

Rintoul, S. R., and C. Wunsch, 1991: Mass, heat, oxygen and nutrient fluxes and budgets in the North Atlantic Ocean. Deep-Sea Res., 38, S355-S377, https://doi.org/10.1016/ S0198-0149(12)80017-3.

Young, W. R., 2012: An exact thickness-weighted average formulation of the Boussinesq equations. J. Phys. Oceanogr., 42, 692-707, https://doi.org/10.1175/JPO-D-11-0102.1. 